\title{
Basic properties of multiplication and composition operators between distinct Orlicz spaces
}

\author{
T. Chawziuk ${ }^{1}$ - Y. Estaremi ${ }^{2}$ - H. Hudzik ${ }^{1,3}$. \\ S. Maghsoudi ${ }^{4}$ - I. Rahmani ${ }^{4}$
}

Received: 31 March 2016 / Accepted: 16 November 2016 / Published online: 15 December 2016 (C) The Author(s) 2016. This article is published with open access at Springerlink.com

\begin{abstract}
First, we present some simple (and easily verifiable) necessary conditions and sufficient conditions for boundedness of the multiplication operator $M_{u}$ and composition operator $C_{T}$ acting from Orlicz space $L^{\Phi_{1}}(\Omega)$ into Orlicz space $L^{\Phi_{2}}(\Omega)$ over arbitrary complete, $\sigma$-finite measure space $(\Omega, \Sigma, \mu)$. Next, we investigate the problem of conditions on the generating Young functions, the function $u$, and/or the function $h=d\left(\mu \circ T^{-1}\right) / d \mu$, under which the operators $M_{u}$ and $C_{T}$ are of closed range or finite rank. Finally, we give necessary and sufficient conditions for boundedness of the operators $M_{u}$ and $C_{T}$ in terms of techniques developed within the theory of Musielak-Orlicz spaces.
\end{abstract}

\footnotetext{
$\bowtie \quad$ H. Hudzik

hudzik@amu.edu.pl

T. Chawziuk

tchawz@gmail.com

Y. Estaremi

yestaremi@pnu.ac.ir

S. Maghsoudi

s_maghsodi@znu.ac.ir

I. Rahmani

i_rahmani@znu.ac.ir

1 Faculty of Mathematics and Computer Science, Adam Mickiewicz University in Poznań, Umultowska 87, 61-614 Poznan, Poland

2 Department of Mathematics, Payame Noor University (PNU), P. O. Box: 19395-3697, Tehran, Iran

3 Faculty of Eonomics and Information Technology, The State University of Applied Sciences in Płock, Nowe Trzepowo 55, 09-402 Płock, Poland

4 Department of Mathematics, University of Zanjan, P.O. Box: 45195-313, Zanjan, Iran
} 
Keywords Multiplication operator - Composition operator - Continuous operators · Closed-range operators · Finite-rank operators · Orlicz space · Musielak-Orlicz space $\cdot$ Lebesgue space $\cdot$ Measurable transformation $\cdot$ Radon-Nikodym derivative

Mathematics Subject Classification 47B38 - 47B33 · 46E30

\section{Introduction}

Given the functions $f$ and $T(f: \Omega \rightarrow \mathbb{R}, T: \Omega \rightarrow \Omega$ ), one way to produce, under certain conditions, a new function is to compose them, i.e. to evaluate $f$ at points $T(t)$. The resulting function is denoted by $f \circ T$, and the operator $f \mapsto f \circ T$, as $f$ runs through a linear function space, is a linear operator $C_{T}$ called the composition operator induced by a mapping $T$. Another way to produce a new function out of given functions $u$ and $f$ (each from $\Omega$ into $\mathbb{R}$ ) is to multiply them, whenever it makes sense, and this gives rise to the operator called the multiplication operator $M_{u}$ induced by a function $u$. A combination of the two methods leads to yet another operator, named the weighted composition operator, defined by $M_{u} C_{T} f=u(f \circ T)$. Composition and multiplication operators received much attention over the past several decades, especially in spaces of measurable functions such as $L^{p}$-spaces, Bergman spaces, and, to a lesser degree, Orlicz spaces. They also play an important role in the study of operators on Hilbert spaces. Consequently, by now there exists a vast literature on the properties of these transformations in various function spaces; we refer the interested reader to the beautiful books $[5,35,36]$, and, for more recent studies, to [9-11,14,23,25], for instance.

As said, composition and multiplication operators between classical $L^{p}$-spaces have been the subject-matter of intensive and extensive study and they feature prominently in operator theory, operator algebras, dynamical systems, and endomorphisms of Banach algebras. Composition operators were used by Banach [2] in his study of isometries between function spaces. In this paper, we aim at a generalization to Orlicz spaces of some results of the paper [38] concerning the classical Lebesgue spaces. Apparently, the paper [24] by Kumar in 1997 was the first attempt at the study of composition operators between Orlicz spaces. In 2004, Cui, Hudzik, Kumar, and Maligranda in [6] considered the composition operator between Orlicz spaces induced by a non-singular measurable transformation and studied its boundedness and compactness. Some other results on the boundedness and compactness of the composition operator between Orlicz spaces were published in $[6-8,12,13,16,21,25,33]$.

Our concern in this paper is to state and prove some necessary conditions, sufficient conditions, and some simultaneously necessary and sufficient conditions for the composition and multiplication operator between distinct Orlicz spaces to be bounded or to have closed-range or finite rank. Our results generalize and improve on some recent results to be found in the literature. 


\section{Preliminaries and basic lemmas}

In this section, for the convenience of the reader, we gather some essential facts on Orlicz spaces and prove two basic lemmas for later use. For more detail on Orlicz spaces, see [22,34].

A function $\Phi: \mathbb{R} \rightarrow[0, \infty]$ is called a Young function if $\Phi$ is convex, even, and $\Phi(0)=0$; we will also assume that $\Phi$ is neither identically zero nor identically infinite on $(0, \infty)$. The fact that $\Phi(0)=0$, along with the convexity of $\Phi$, implies that $\lim _{x \rightarrow 0^{+}} \Phi(x)=0$; while $\Phi \neq 0$, again along with the convexity of $\Phi$, implies that $\lim _{x \rightarrow \infty} \Phi(x)=\infty$. We set $a_{\Phi}:=\sup \{x \geq 0: \Phi(x)=0\}$ and $b_{\Phi}:=\sup \{x>0$ : $\Phi(x)<\infty\}$. Then it can be checked that $\Phi$ is continuous and nondecreasing on $\left[0, b_{\Phi}\right)$ and strictly increasing on $\left[a_{\Phi}, b_{\Phi}\right)$ if $a_{\Phi}<b_{\Phi}$. We also assume the left-continuity of the function $\Phi$ at $b_{\Phi}$, i.e. $\lim _{x \rightarrow b_{\Phi}^{-}} \Phi(x)=\Phi\left(b_{\Phi}\right) \in[0, \infty]$.

To each Young function $\Phi$ is associated another convex function $\Psi: \mathbb{R} \rightarrow[0, \infty)$ with similar properties, defined by

$$
\Psi(y)=\sup \{x|y|-\Phi(x): x \geq 0\} \quad(y \in \mathbb{R}) .
$$

The function $\Psi$ is called the function complementary to $\Phi$ in the sense of Young. Any pair of complementary functions $(\Phi, \Psi)$ satisfies Young's inequality $x y \leq \Phi(x)+$ $\Psi(y)(x, y \in R)$.

The generalized inverse of the Young function $\Phi$ is defined by

$$
\Phi^{-1}(y)=\inf \{x \geq 0: \Phi(x)>y\} \quad(y \in[0, \infty)) .
$$

Notice that if $x \geq 0$, then $\Phi\left(\Phi^{-1}(x)\right) \leq x$, and if $\Phi(x)<\infty$, we also have $x \leq$ $\Phi^{-1}(\Phi(x))$. There are equalities in both cases when $\Phi$ is a Young function vanishing only at zero and taking only finite values. Also, if $(\Phi, \Psi)$ is a pair of complementary Young functions, then

$$
x<\Phi^{-1}(x) \Psi^{-1}(x) \leq 2 x
$$

for all $x \geq 0$ (Proposition 2.1.1(ii) in [34]).

By an $N$-function we mean a Young function vanishing only at zero, taking only finite values, and such that $\lim _{x \rightarrow \infty} \Phi(x) / x=\infty$ and $\lim _{x \rightarrow 0^{+}} \Phi(x) / x=0$. Note that then $a_{\Phi}=0, b_{\Phi}=\infty$, and, as we said above, $\Phi$ is continuous and strictly increasing on $[0, \infty)$. Moreover, a function complementary to an $N$-function is again an $N$-function.

A Young function $\Phi$ is said to satisfy the $\Delta_{2}$-condition at $\infty$ if $\Phi(2 x) \leq K \Phi(x)(x \geq$ $x_{0}$ ) for some constants $K>0$ and $x_{0}>0$ with $\Phi\left(x_{0}\right)<\infty$. A Young function $\Phi$ satisfies the $\Delta_{2}$-condition globally if $\Phi(2 x) \leq K \Phi(x)(x \geq 0)$ for some $K>0$.

A Young function $\Phi$ is said to satisfy the $\Delta^{\prime}$-condition (respectively, the $\nabla^{\prime}$ condition) at $\infty$, if there exist $c>0$ (respectively, $b>0$ ) and $x_{0}>0$ with $\Phi\left(x_{0}\right)<\infty$ such that

$$
\begin{aligned}
\Phi(x y) & \leq c \Phi(x) \Phi(y) \quad\left(x, y \geq x_{0}\right) \\
\text { (respectively, } \Phi(b x y) & \left.\geq \Phi(x) \Phi(y) \quad\left(x, y \geq x_{0}\right)\right) .
\end{aligned}
$$


If $x_{0}=0$, these conditions are said to hold globally. Notice that if $\Phi \in \Delta^{\prime}$, then $\Phi \in \Delta_{2}$ (both at $\infty$ and globally).

Let $\Phi_{1}, \Phi_{2}$ be Young functions. Then $\Phi_{1}$ is called stronger than $\Phi_{2}$ at $\infty$, which is denoted by $\Phi_{1} \stackrel{\ell}{\succ} \Phi_{2}\left[\right.$ or $\Phi_{2} \stackrel{\ell}{\prec} \Phi_{1}$ ], if

$$
\Phi_{2}(x) \leq \Phi_{1}(a x) \quad\left(x \geq x_{0}\right)
$$

for some $a \geq 0$ and $x_{0}>0$ with $\Phi_{2}\left(x_{0}\right)<\infty$; if $x_{0}=0$, this condition is said to hold globally and is then denoted by $\Phi_{1} \stackrel{a}{\succ} \Phi_{2}$ [or $\Phi_{2} \stackrel{a}{\prec} \Phi_{1}$ ]. We record the following observation for later use.

Lemma 2.1 If $\Phi_{1}, \Phi_{2}, \Phi_{3}$ are Young functions vanishing only at zero, taking only finite values, and such that

$$
\Phi_{1}(x y) \leq \Phi_{2}(x)+\Phi_{3}(y) \quad(x, y \geq 0),
$$

then $\Phi_{2} \stackrel{\ell}{\nprec} \Phi_{1}$, and hence also $\Phi_{2} \stackrel{a}{\nprec} \Phi_{1}$.

Proof The inequality in the assumptions of the lemma implies that $\Phi_{1}\left(\Phi_{2}^{-1}(x) \Phi_{3}^{-1}(x)\right)$ $\leq 2 x$ for all $x \geq 0$, and this, together with subadditivity of $\Phi_{1}^{-1}$, leads to

$$
\Phi_{2}^{-1}(x) \Phi_{3}^{-1}(x) \leq \Phi_{1}^{-1}(2 x) \leq 2 \Phi_{1}^{-1}(x) \quad(x \geq 0) .
$$

Now suppose, towards a contradiction, that there are $a>0$ and $x_{0}>0$ such that

$$
\Phi_{2}(x)<\Phi_{1}(a x) \quad\left(x \geq x_{0}\right) .
$$

Then, since $\Phi_{1}^{-1}$ is increasing on $[0, \infty)$, we have $\Phi_{1}^{-1}\left(\Phi_{2}(x)\right)<\Phi_{1}^{-1}\left(\Phi_{1}(a x)\right)=$ $a x$, and so, replacing $x$ with $\Phi_{2}(x)$ in (2.2), we get

$$
x \Phi_{3}^{-1}\left(\Phi_{2}(x)\right)=\Phi_{2}^{-1}\left(\Phi_{2}(x)\right) \Phi_{3}^{-1}\left(\Phi_{2}(x)\right) \leq 2 \Phi_{1}^{-1}\left(\Phi_{2}(x)\right)<2 a x \quad\left(x \geq x_{0}\right) .
$$

This means, since $\Phi_{3}$ is increasing on $[0, \infty)$, that $\Phi_{1}(x)<\Phi_{3}(2 a)=$ const. for all $x \geq x_{0}$, a contradiction, because $\Phi_{2}(x) \rightarrow \infty$ as $x \rightarrow \infty$.

Let $(\Omega, \Sigma, \mu)$ be a complete $\sigma$-finite measure space and let $L^{0}(\Omega)$ be the linear space of equivalence classes of $\Sigma$-measurable real-valued functions on $\Omega$, that is, we identify functions equal $\mu$-almost everywhere on $\Omega$. The support $S$ of a measurable function $f$ is defined by $S(f):=\{t \in \Omega: f(t) \neq 0\}$. For a Young function $\Phi$, the space

$$
L^{\Phi}(\Omega)=\left\{f \in L^{0}(\Omega): \exists k>0, I_{\Phi}(k f)<\infty\right\}
$$

is a Banach space if it is equipped with the norm

$$
\|f\|_{\Phi}=\inf \left\{k>0: I_{\Phi}(f / k) \leq 1\right\}
$$

where $I_{\Phi}(f)=\int_{\Omega} \Phi(f(t)) d \mu(t)$. The couple $\left(L^{\Phi}(\Omega),\|\cdot\|_{\Phi}\right)$ is called the Orlicz space generated by a Young function $\Phi$. For any $f \in L^{0}(\Omega)$, we have $I_{\Phi}(f) \leq 1$ if 
and only if $\|f\|_{\Phi} \leq 1$. In particular, if $I_{\Phi}(f) \leq a(a>0)$, then $\|f\|_{\Phi} \leq \max (a, 1)$. If $\Phi \in \Delta_{2}$ and $f \in L^{\Phi}(\Omega)$, then $I_{\Phi}(k f)<\infty$ for all $k>0$.

Let $\Phi(x)=|x|^{p} / p$ with $1<p<\infty$; $\Phi$ is then a Young function and $\Psi(x)=$ $|x|^{p^{\prime}} / p^{\prime}$, with $1 / p+1 / p^{\prime}=1$, is the Young function complementary to $\Phi$. Thus, with this function $\Phi$ we retrieve the classical Lebesgue space $L^{p}(\Omega)$, i.e. $L^{\Phi}(\Omega)=L^{p}(\Omega)$.

Recall that an atom of the measure space $(\Omega, \Sigma, \mu)$ is a set $\mathfrak{A} \in \Sigma$ with $\mu(\mathfrak{A})>0$ such that if $F \in \Sigma$ and $F \subset \mathfrak{A}$, then either $\mu(F)=0$ or $\mu(F)=\mu(\mathfrak{A})$. A measure space $(\Omega, \Sigma, \mu)$ with no atoms is called a non-atomic measure space [29].

Remark 2.2 By saying that $\Phi \in \Delta_{2}$ we will always mean that the Young function $\Phi$ satisfies the $\Delta_{2}$-condition globally, since we place no restrictions on the underlying measure space $(\Omega, \Sigma, \mu)$, which can be finite or infinite, non-atomic, purely atomic, or mixed.

The following lemma is a key tool in some of our investigations.

Lemma 2.3 Let $\Phi_{1}, \Phi_{2}$ be Young functions such that $\Phi_{2} \stackrel{\ell}{\nprec} \Phi_{1}$. If $E$ is a non-atomic $\Sigma$-measurable set with positive measure, then there exists $f \in L^{\Phi_{1}}(\Omega)$ such that $f_{\left.\right|_{E}} \notin L^{\Phi_{2}}(E)$.

Proof It is well-known that if $L^{\Phi_{1}}(\Omega, \Sigma, \mu) \subset L^{\Phi_{2}}(\Omega, \Sigma, \mu)$ for a non-atomic measure space $(\Omega, \Sigma, \mu)$, then $\Phi_{2} \stackrel{\ell}{\prec} \Phi_{1}$ (see, for instance, [26] Theorem 3.4, and $[28,30,31]$ for some extensions). Therefore, if $\Phi_{2} \stackrel{\ell}{\nprec} \Phi_{1}$ and $E$ is a $\Sigma$-measurable subset of $\Omega$ with $\mu(E)>0$, then we can find a function $g \in L^{\Phi_{1}}(E)$ such that $g \notin L^{\Phi_{2}}(E)$. Defining $f(t)=g(t)$ for $t \in E$, and $f(t)=0$ for $t \notin E$, we have the desired function.

Remark 2.4 We will use Lemmas 2.1 and 2.3 in conjunction since the thesis of the former is the assumption of the latter.

Given a measurable function $u \in L_{+}^{0}(\Omega)$ the mapping $f \mapsto u f$ is a linear transformation on $L^{0}(\Omega)$ called the multiplication operator induced by $u$ and denoted $M_{u}$.

Let $T: \Omega \rightarrow \Omega$ be a measurable transformation. The transformation $T$ is said to be non-singular if $\mu\left(T^{-1}(A)\right)=0$ for all $A \in \Sigma \cap T(\Omega)$ with $\mu(A)=0$. The condition ensures that the measure $\mu \circ T^{-1}$ defined by $\mu \circ T^{-1}(A)=\mu\left(T^{-1}(A)\right)$ for all $A \in \Sigma \cap T(\Omega)$ is absolutely continuous with respect to the measure $\mu$ (this fact is usually denoted by $\mu \circ T^{-1} \ll \mu$ ). Then the Radon-Nikodym theorem guarantees the existence of a non-negative locally integrable function $h: T(\Omega) \rightarrow \mathbb{R}_{+}$, called the Radon-Nikodym derivative of $\mu \circ T^{-1}$ with respect to $\mu$, such that

$$
\mu \circ T^{-1}(A)=\int_{A} h d \mu \quad(A \in \Sigma \cap T(\Omega)) .
$$

We do not necessarily assume that the transformation $T$ is surjective or injective.

The non-singular measurable transformation $T$ induces a linear operator $C_{T}$ on $L^{0}(\Omega)$, called the composition operator, defined by

$$
C_{T} f(t)=f(T(t)) \quad\left(t \in \Omega, f \in L^{0}(\Omega)\right) .
$$


Finally, we recall two well-known facts from measure theory; for proofs see, for example, Section 11.9 in [29]:

(i) If $(\Omega, \Sigma, \mu)$ be a $\sigma$-finite measure space, then for every measurable real-valued function $f$ on $\Omega$ and every atom $\mathfrak{A}$, there is a real number, denoted by $f(\mathfrak{A})$, such that $f=f(\mathfrak{A}) \mu$-a.e. on $\mathfrak{A}$.

(ii) If $(\Omega, \Sigma, \mu)$ is a $\sigma$-finite measure space that fails to be non-atomic, there is a non-empty countable set of pairwise disjoint atoms $\left\{\mathfrak{A}_{n}\right\}_{n \in \mathbb{N}}$ with the property that $\mathfrak{B}:=\Omega \backslash \bigcup_{n \in \mathbb{N}} \mathfrak{A}_{n}$ contains no atoms. We assume that both the atoms and their counterimages under $T$ have strictly positive measure. The latter fact means that the Radon-Nikodym derivative $h$ is strictly positive on all atoms from $T(\Omega)$.

We keep the above notation throughout the paper.

\section{Boundedness of the multiplication operator}

In this section we state various necessary conditions and sufficient conditions under which the multiplication operator $M_{u}$ between distinct Orlicz spaces is bounded.

Recall the well-known theorem due to O'Neil [32] on the multiplication operator between Orlicz spaces (see also [1] and Theorem 5.4.1 in [33]): suppose that $\Phi_{1}, \Phi_{2}$, $\Phi_{3}$ are Young functions such that either (a) there exist $c>0$ and $x_{0}>0$ with $\Phi_{2}\left(x_{0}\right)<$ $\infty$ such that $\Phi_{2}(c x y) \leq \Phi_{1}(x)+\Phi_{3}(y)$ for all $x, y \geq x_{0}$ in the case when $\mu(\Omega)<\infty$ or (b) there exists $c>0$ such that $\Phi_{2}(c x y) \leq \Phi_{1}(x)+\Phi_{3}(y)$ for all $x, y>0$ in the case when $\mu(\Omega)=\infty$. Let $\Omega$ contain a non-atomic set of positive measure. Then, whenever $u \in L^{\Phi_{3}}(\Omega)$, the multiplication operator $M_{u}$ is bounded from $L^{\Phi_{1}}(\Omega)$ into $L^{\Phi_{2}}(\Omega)$.

Before going any further, let us consider some related examples.

Example 3.1 (i) Let $\Phi$ and $\Psi$ be complementary Young functions. Since $\frac{|x|^{p}}{p} \leq$ $\frac{1}{p}\left(\Phi(x)+\Psi\left(x^{p-1}\right)\right)$ and $\frac{|x|^{p}}{p} \leq \frac{1}{p}\left(\Phi\left(x^{p-1}\right)+\Psi(x)\right)$, for $x \geq 0$ and $p>2$, by O'Neil's theorem we get that if $u^{\frac{1}{p-1}} \in L^{\Psi}(\Omega)$ then the operator $M_{u}$ is bounded from $L^{\Phi}(\Omega)$ into $L^{p}(\Omega)$, and if $v^{\frac{1}{p-1}} \in L^{\Phi}(\Omega)$ then the operator $M_{v}$ is bounded from $L^{\Psi}(\Omega)$ into $L^{p}(\Omega)$.

(ii) Let $\Omega=[a, b], a, b>1, p>1$, and let $\mu$ be Lebesgue measure. If we take $\Phi_{1}(x)=e^{|x|^{p}}-|x|^{p}-1, \Phi_{2}(x)=\frac{|x|^{p}}{p}$, and $\Phi_{3}(x)=\left(1+|x|^{p}\right) \log (1+$ $\left.|x|^{p}\right)-|x|^{p}$, then, since $\phi_{1}(x)=\Phi_{1}\left(|x|^{1 / p}\right)$ and $\phi_{3}(x)=\Phi_{3}\left(|x|^{1 / p}\right)$ are complementary Young functions, Young's inequality yields $\Phi_{2}(x y) \leq \Phi_{1}(x)+\Phi_{3}(y)$ for all $x, y \geq 0$. Now, if $u(t)=\sqrt[p]{t^{p}-1}$, it is clear that $I_{\Phi_{3}}(u)<\infty$, i.e. $u \in L^{\Phi_{3}}(\Omega)$. O'Neil's theorem implies that $M_{u}$ is a bounded operator from $L^{\Phi_{1}}(\Omega)$ into $L^{\Phi_{2}}(\Omega)$.

(iii) Let $A=(0, a], B=\{\ln t: t \in \mathbb{N}, t>a\}$, for $a>0$, and let $\Omega=A \cup B$. Take $\Phi_{1}(x)=e^{|x|}-|x|-1, \Phi_{2}(x)=(1+|x|) \ln (1+|x|)-|x|$, and for every Lebesgue measurable $C \subset \Omega$ define $\mu(C)=\mu_{1}(C \cap A)+\mu_{2}(C \cap B)$, where $\mu_{1}$ is Lebesgue measure and $\mu_{2}(\{\ln t\})=1 / t^{3}$ for $\ln t \in B$. If we set $u(t)=1 / t^{2}$, then $M_{u}$ is not bounded from $L^{\Phi_{1}}(\Omega)$ into $L^{\Phi_{2}}(\Omega)$. Indeed, for $f(t)=t$ we 
have

$$
\begin{aligned}
I_{\Phi_{1}}(f) & =\int_{\Omega}\left(\Phi_{1} \circ f\right) d \mu=\int_{\Omega}\left(e^{t}-t-1\right) d \mu \\
& =\int_{A}\left(e^{t}-t-1\right) d \mu+\int_{B}\left(e^{t}-t-1\right) d \mu<\infty,
\end{aligned}
$$

where we used the following calculation

$$
\int_{B}\left(e^{t}-t-1\right) d \mu=\sum_{n>a}\left(e^{\ln n}-\ln n-1\right) \frac{1}{n^{3}}<\sum_{n>a} \frac{1}{n^{2}}<\infty .
$$

Hence $f \in L^{\Phi_{1}}(\Omega)$. But for any $\lambda>0$ we have

$$
\begin{aligned}
I_{\Phi_{2}}\left(\lambda M_{u} f\right)= & \int_{\Omega}\left(\Phi_{2} \circ \lambda M_{u} f\right) d \mu=\int_{\Omega} \Phi_{2}\left(\frac{\lambda}{t}\right) d \mu \\
= & \int_{\Omega}\left(\left(1+\frac{\lambda}{t}\right) \ln \left(1+\frac{\lambda}{t}\right)-\frac{\lambda}{t}\right) d \mu \\
= & \int_{A}\left(\left(1+\frac{\lambda}{t}\right) \ln \left(1+\frac{\lambda}{t}\right)-\frac{\lambda}{t}\right) d \mu \\
& +\int_{B}\left(\left(1+\frac{\lambda}{t}\right) \ln \left(1+\frac{\lambda}{t}\right)-\frac{\lambda}{t}\right) d \mu \\
> & \int_{0}^{a}\left(\left(1+\frac{\lambda}{t}\right) \ln \left(1+\frac{\lambda}{t}\right)-\frac{\lambda}{t}\right) d t \\
= & \lambda \int_{\lambda / a}^{\infty}((1+s) \ln (1+s)-s) \frac{1}{s^{2}} d s \\
= & \lambda \int_{\lambda / a}^{\infty}\left(\frac{\ln (1+s)}{s^{2}}+\frac{\ln (1+s)-1}{s}\right) d s \\
> & \lambda \int_{\lambda / a}^{\infty}\left(\frac{\ln (1+s)-1}{s}\right) d s=\infty,
\end{aligned}
$$

and so $M_{u} f \notin L^{\Phi_{2}}(\Omega)$. The operator $M_{u}$ does even act from $L^{\Phi_{1}}(\Omega)$ into $L^{\Phi_{2}}(\Omega)$ and, by O'Neil's theorem, we conclude that if a Young function $\Phi_{3}$ satisfies $\Phi_{2}(x y) \leq \Phi_{1}(x)+\Phi_{3}(y)$ for all $x, y \geq 0$, then $u$ does not belong to $L^{\Phi_{3}}(\Omega)$.

Maligranda and Persson in [27] proved a theorem which can be stated in the following way: assume that $\Phi_{1}, \Phi_{2}, \Phi_{3}$ are Young functions with values in $[0, \infty)$ such that $\Phi_{2}(x y) \leq \Phi_{1}(x)+\Phi_{3}(y)$ for all $x, y \geq 0$ and $\Phi_{2}^{-1}(x) \leq \Phi_{1}^{-1}(x) \Phi_{3}^{-1}(x)$ for all $x \geq 0$, and assume further that either (A) $\Omega$ is a non-atomic measure space or (B) $\Phi_{2}(x)=x^{r}$ and $x^{-r} \Phi_{1}(x)$ is nondecreasing. Then $M_{u}$ is a bounded multiplication operator from $L^{\Phi_{1}}(\Omega)$ into $L^{\Phi_{2}}(\Omega)$ if and only if $u \in L^{\Phi_{3}}(\Omega)$.

These results have been generalized and extended to the more general setting of Calderón-Lozanovskiı̌ spaces in two recent papers $[19,20]$ by Kolwicz et al. 
We give another necessary condition on the function $u$ so that it induces a bounded multiplication operator $M_{u}$ between distinct Orlicz spaces; it will be used in the proof of Theorem 4.11.

Proposition 3.2 Let $\Phi_{1}$ and $\Phi_{2}$ be Young functions vanishing only at zero, taking only finite values, and such that $\Phi_{1}$ is an $N$-function, $\Phi_{1} \in \Delta^{\prime}, \Phi_{2} \in \Delta_{2}$, and $\Phi_{3}:=\Psi_{2} \circ \Psi_{1}^{-1}$ is a Young function. If $u \in L_{+}^{0}(\Omega)$ and $M_{u}$ is a bounded multiplication operator from $L^{\Phi_{1}}(\Omega)$ into $L^{\Phi_{2}}(\Omega)$, then $u \in L^{\Psi_{3} \circ \Psi_{1}}(\Omega)$.

Proof Let $u \in L_{+}^{0}(\Omega)$ and let $M_{u}$ be a bounded multiplication operator from $L^{\Phi_{1}}(\Omega)$ into $L^{\Phi_{2}}(\Omega)$ induced by the function $u$. Since $\Phi_{1} \in \Delta_{2}$ (as a consequence of the fact that $\left.\Phi_{1} \in \Delta^{\prime}\right)$ and $\Phi_{2} \in \Delta_{2}$, the dual spaces of $L^{\Phi_{1}}(\Omega)$ and $L^{\Phi_{2}}(\Omega)$ are equal to $L^{\Psi_{1}}(\Omega)$ and $L^{\Psi_{2}}(\Omega)$, respectively. Hence the adjoint operator $M_{u}^{*}$ to the bounded operator $M_{u}$ is bounded and acts from $L^{\Psi_{2}}(\Omega)$ into $L^{\Psi_{1}}(\Omega)$.

It is easy to check that $\Phi_{1} \in \Delta^{\prime}$ implies that $\Psi_{1} \in \nabla^{\prime}$, i.e. there is $b>0$ such that $\Psi_{1}(b x y) \geq \Psi_{1}(x) \Psi_{1}(y)$ for all $x, y \geq 0$.

Let $f$ be any function from $L^{\Phi_{3}}(\Omega)$. By the definition of $\Phi_{3}$, we have that the function $\Psi_{1}^{-1} \circ|f|$ is contained in the space $L^{\Psi_{2}}(\Omega)$, and so $M_{u}^{*}\left(\Psi_{1}^{-1} \circ|f|\right)$ is contained in the space $L^{\Psi_{1}}(\Omega)$. Therefore, there is $\lambda>0$ such that $I_{\Psi_{1}}\left(\lambda M_{u}^{*}\left(\Psi_{1}^{-1} \circ|f|\right)\right)<\infty$. We have

$$
\begin{aligned}
\left|\int_{\Omega}\left(\Psi_{1} \circ \frac{\lambda}{b} u\right) f d \mu\right| & \leq \int_{\Omega}\left(\Psi_{1} \circ \frac{\lambda}{b} u\right)|f| d \mu \leq \int_{\Omega}\left(\Psi_{1} \circ \frac{\lambda}{b} u\right)\left(\Psi_{1} \circ \Psi_{1}^{-1} \circ|f|\right) d \mu \\
& \leq \int_{\Omega} \Psi_{1}\left(b \frac{\lambda}{b} u \Psi_{1}^{-1} \circ|f|\right) d \mu=\int_{\Omega} \Psi_{1}\left(\lambda M_{u}^{*}\left(\Psi_{1}^{-1} \circ|f|\right)\right) d \mu<\infty .
\end{aligned}
$$

This means that $\Psi_{1} \circ \frac{\lambda}{b} u$ belongs to the Köthe dual of $L^{\Phi_{3}}(\Omega)$, which is equal to $L^{\Psi_{3}}(\Omega)$. Consequently, there is $\alpha \in(0,1)$ such that $I_{\Psi_{3}}\left(\alpha \Psi_{1} \circ \frac{\lambda}{b} u\right)<\infty$, whence $I_{\Psi_{3} \circ \Psi_{1}}\left(\alpha \frac{\lambda}{b} u\right)=I_{\Psi_{3}}\left(\Psi_{1} \circ \alpha \frac{\lambda}{b} u\right) \leq I_{\Psi_{3}}\left(\alpha \Psi_{1} \circ \frac{\lambda}{b} u\right)<\infty$. Hence $u \in L^{\Psi_{3} \circ \Psi_{1}}(\Omega)$.

In the case of a non-atomic complete and $\sigma$-finite measure space, some necessary and sufficient conditions for the boundedness of $M_{u}$ and $C_{T}$ from $L^{\Phi_{1}}(\Omega)$ into $L^{\Phi_{2}}(\Omega)$ were established in [3]. It turns out that these are equivalent to the conditions for the inclusion of the Orlicz space $L^{\Phi_{1}}(\Omega)$ into the Musielak-Orlicz space $L^{\Phi_{2}, u}(\Omega)$ and of the Orlicz space $L^{\Phi_{1}}(T(\Omega))$ into the Musielak-Orlicz space $L_{h}^{\Phi_{2}}(T(\Omega)$ ), where $h=d\left(\mu \circ T^{-1}\right) / d \mu$, respectively. The spaces $L^{\Phi_{2}, u}(\Omega)$ and $L_{h}^{\Phi_{2}}(T(\Omega))$ are generated over the measure spaces $(\Omega, \Sigma, \mu)$ and $\left(T(\Omega), \Sigma \cap T(\Omega), \mu_{\mid \Sigma \cap T(\Omega)}\right)$ by the MusielakOrlicz functions $\Phi_{2}(x u(t))(t \in \Omega$ and $x \in \mathbb{R})$ and $\Phi_{2}(x) h(t)(t \in T(\Omega)$ and $x \in \mathbb{R})$, respectively, $t \in \Omega$ and $x \in \mathbb{R}$.

We shall now prove a theorem from which it follows that, if the measure space $(\Omega, \Sigma, \mu)$ is non-atomic, for the above inclusions to hold it is necessary that $\Phi_{2} \prec \Phi_{1}$. This might come as a surprise since it means that the simple condition $\Phi_{2} \prec \Phi_{1}$, which does not involve the Radon-Nikodym derivative $h$, is necessary for the inclusion $L^{\Phi_{1}}(T(\Omega)) \subset L_{h}^{\Phi_{2}}(T(\Omega))$, which does. 
Theorem 3.3 Let $\Phi_{1}, \Phi_{2}$ be Young functions such that $\Phi_{2} \stackrel{\ell}{\nprec} \Phi_{1}$ and let $(\Omega, \Sigma, \mu)$ be a non-atomic measure space. Then there are no non-zero bounded operators $C_{T} M_{u}$ from $L^{\Phi_{1}}(\Omega)$ into $L^{\Phi_{2}}(\Omega)$.

Proof Suppose, to the contrary, that $C_{T} M_{u}$ is a non-zero bounded linear operator from $L^{\Phi_{1}}(\Omega)$ into $L^{\Phi_{2}}(\Omega)$ and let

$$
E_{n}=\left\{t \in \Omega: u(t)>\frac{1}{n}\right\} \bigcap\left\{t \in T(\Omega): h(t)>\frac{1}{n}\right\}
$$

For any $n \in \mathbb{N}$. Then $\left\{E_{n}\right\}_{n \in \mathbb{N}}$ is an increasing sequence of $\Sigma$-measurable sets. Since $C_{T} M_{u}$ is non-zero, $\mu\left(E_{m}\right)>0$ for some $m \in \mathbb{N}$, whence also $\mu\left(E_{n}\right)>0$ for all $n \geq m$. We assume without loss of generality that $\mu\left(E_{n}\right)>0$ for all $n \in \mathbb{N}$. Let $F \subset E:=\bigcup_{n} E_{n}$ and $0<\mu(F)<\infty$. The assumption that $\Phi_{2} \stackrel{\ell}{\nprec} \Phi_{1}$ implies that an increasing sequence of positive numbers $\left\{y_{n}\right\}$ can be found such that $\Phi_{2}\left(y_{n}\right)>$ $\Phi_{1}\left(2^{n} n^{3} y_{n}\right)$. Since the measure space $(\Omega, \Sigma, \mu)$ is non-atomic, we can choose a sequence $\left\{F_{n}\right\}$ of pairwise disjoint measurable subsets of $F$ such that $F_{n} \subset E_{n}$ and $\mu\left(F_{n}\right)=\frac{\Phi_{1}\left(y_{1}\right) \mu(F)}{2^{n} \Phi_{1}\left(n^{3} y_{n}\right)}$ For any $n \in \mathbb{N}$. This is possible because $v$ is non-atomic and $\Phi_{1}\left(n^{3} y_{n}\right) \geq \Phi_{1}\left(y_{n}\right) \geq \Phi_{1}\left(y_{1}\right)$, and so $\mu\left(F_{n}\right) \leq \frac{\mu(F)}{2^{n}}$, and $\sum_{n=1}^{\infty} \frac{\mu(F)}{2^{n}}=\mu(F)$.

Define the function $f:=\sum_{n=1}^{\infty} b_{n} \chi_{F_{n}}$, where $b_{n}:=n^{2} y_{n}$, and take arbitrary $\alpha>0$. Then for a natural number $n_{0}>\alpha$ we have

$$
\begin{aligned}
I_{\Phi_{1}}(\alpha f) & =\int_{\Omega} \Phi_{1}(\alpha f) d \mu=\sum_{n=1}^{\infty} \int_{\Omega} \Phi_{1}\left(\alpha b_{n}\right) \chi_{F_{n}} d \mu \\
& =\sum_{n=1}^{n_{0}} \Phi_{1}\left(\alpha b_{n}\right) \mu\left(F_{n}\right)+\sum_{n>n_{0}} \Phi_{1}\left(\alpha b_{n}\right) \mu\left(F_{n}\right) \\
& =\sum_{n=1}^{n_{0}} \Phi_{1}\left(\alpha b_{n}\right) \mu\left(F_{n}\right)+\mu(F) \sum_{n>n_{0}} \frac{\Phi_{1}\left(\alpha b_{n}\right) \Phi_{1}\left(y_{1}\right)}{2^{n} \Phi_{1}\left(n^{3} y_{n}\right)} \\
& \leq \sum_{n=1}^{n_{0}} \Phi_{1}\left(\alpha b_{n}\right) \mu\left(F_{n}\right)+\mu(F) \sum_{n>n_{0}} \frac{\Phi_{1}\left(n^{3} y_{n}\right) \Phi_{1}\left(y_{1}\right)}{2^{n} \Phi_{1}\left(n^{3} y_{n}\right)}<\infty .
\end{aligned}
$$

This implies that $f \in L^{\Phi_{1}}(\Omega)$. But for $m_{0}>0$ such that $\frac{1}{m_{0}}<\alpha$, we obtain

$$
\begin{aligned}
I_{\Phi_{2}}\left(\alpha C_{T} M_{u} f\right) & =\int_{\Omega} \Phi_{2}\left(\alpha C_{T} M_{u} f\right) d \mu=\int_{T(\Omega)} h(t) \Phi_{2}(\alpha u f) d \mu \\
& \geq \sum_{n \geq m_{0}} \int_{F_{n}} h(t) \Phi_{2}\left(\alpha b_{n} u\right) d \mu \geq \sum_{n \geq m_{0}} \int_{F_{n}} \frac{1}{n} \Phi_{2}\left(b_{n} / n^{2}\right) d \mu \\
& \geq \sum_{n \geq m_{0}} \int_{F_{n}} \frac{1}{n} \Phi_{2}\left(y_{n}\right) d \mu \geq \sum_{n \geq m_{0}} \frac{1}{n} \Phi_{1}\left(2^{n} n^{3} y_{n}\right) \mu\left(F_{n}\right) \\
& \geq \sum_{n \geq m_{0}} \frac{1}{n} 2^{n} \Phi_{1}\left(n^{3} y_{n}\right) \mu\left(F_{n}\right) \geq \mu(F) \sum_{n \geq m_{0}} \frac{1}{n} \Phi_{1}\left(y_{1}\right)=\infty,
\end{aligned}
$$


which, by the arbitratiness of $\alpha>0$, contradicts the boundedness of $C_{T} M_{u}$. (In fact, we even proved that $C_{T} M_{u}$ does not act from $L^{\Phi_{1}}(\Omega)$ into $L^{\Phi_{2}}(\Omega)$ ).

We provide some necessary conditions for the boundedness of the multiplication operator $M_{u}$ from $L^{\Phi_{1}}(\Omega)$ into $L^{\Phi_{2}}(\Omega)$ under the assumption that $\Phi_{1}(x y) \leq \Phi_{2}(x)+$ $\Phi_{3}(y)$ for some Young function $\Phi_{3}$ and for all $x, y \geq 0$.

Theorem 3.4 Let $\Phi_{1}, \Phi_{2}, \Phi_{3}$ be Young functions vanishing only at zero, taking only finite values, and such that $\Phi_{1}(x y) \leq \Phi_{2}(x)+\Phi_{3}(y)$ for all $x, y \geq 0$. If $u \in L_{+}^{0}(\Omega)$ induces a bounded multiplication operator $M_{u}: L^{\Phi_{1}}(\Omega) \rightarrow L^{\Phi_{2}}(\Omega)$, then

(i) $u(t)=0$ for $\mu$-a.e. $t \in \mathfrak{B}$, the non-atomic part of $\Omega$;

(ii) $\sup _{n \in \mathbb{N}} u\left(\mathfrak{A}_{n}\right) \Phi_{3}^{-1}\left(\frac{1}{\mu\left(\mathfrak{A}_{n}\right)}\right)<\infty$.

Proof Let the assumptions about $\Phi_{i}(\mathrm{i}=1,2,3)$ be satisfied and $M_{u}$ be bounded. First we prove (i). If $\mu\{t \in \mathfrak{B}: u(t) \neq 0\}>0$, then there exists a constant $\delta>0$ such that the set $E=\{t \in \mathfrak{B}: u(t)>\delta\}$ has positive measure. Since $E$ is non-atomic, $\mu(E)>0$, and $\Phi_{1}(x y) \leq \Phi_{2}(x)+\Phi_{3}(y)$ for all $x, y \geq 0$, by Lemmas 2.1 and 2.3 we have that there exists $f \in L^{\Phi_{1}}(\Omega)$ such that $f_{\left.\right|_{E}} \notin L^{\Phi_{2}}(E)$, and so

$$
\infty=\int_{E} \Phi_{2}\left(\frac{\delta f(t)}{\left\|M_{u} f\right\|_{\Phi_{2}}}\right) d \mu \leq \int_{\Omega} \Phi_{2}\left(\frac{u(t) f(t)}{\left\|M_{u} f\right\|_{\Phi_{2}}}\right) d \mu \leq 1,
$$

which is a contradiction. Thus (i) holds.

Now we prove (ii). We may assume that the function $u$ is not identically zero. For each $n \in \mathbb{N}$, put $f_{n}=\Phi_{1}^{-1}\left(\frac{1}{\mu\left(\mathfrak{A}_{n}\right)}\right) \chi_{\mathfrak{A}_{n}}$. It is clear that $f_{n} \in L^{\Phi_{1}}(\Omega)$ and $I_{\Phi_{1}}\left(f_{n}\right)=1$, whence $\left\|f_{n}\right\|_{\Phi_{1}}=1$. Since the operator $M_{u}$ is bounded, we have

$$
\begin{aligned}
1 \geq \int_{\Omega} \Phi_{2}\left(\frac{u(t) f_{n}(t)}{\left\|M_{u} f_{n}\right\|_{\Phi_{2}}}\right) d \mu & =\int_{\mathfrak{A}_{n}} \Phi_{2}\left(\frac{u(t) \Phi_{1}^{-1}\left(\frac{1}{\mu\left(\mathfrak{A}_{n}\right)}\right)}{\left\|M_{u} f_{n}\right\|_{\Phi_{2}}}\right) d \mu \\
& =\Phi_{2}\left(\frac{u\left(\mathfrak{A}_{n}\right) \Phi_{1}^{-1}\left(\frac{1}{\mu\left(\mathfrak{A}_{n}\right)}\right)}{\left\|M_{u} f_{n}\right\|_{\Phi_{2}}}\right) \mu\left(\mathfrak{A}_{n}\right) .
\end{aligned}
$$

Therefore

$$
\frac{u\left(\mathfrak{A}_{n}\right) \Phi_{1}^{-1}\left(\frac{1}{\mu\left(\mathfrak{A}_{n}\right)}\right)}{\Phi_{2}^{-1}\left(1 / \mu\left(\mathfrak{A}_{n}\right)\right)} \leq\left\|M_{u} f_{n}\right\|_{\Phi_{2}}
$$

Put $x=1 / \mu\left(\mathfrak{A}_{n}\right)$ in the inequality $\Phi_{2}^{-1}(x) \Phi_{3}^{-1}(x) \leq 2 \Phi_{1}^{-1}(x)$ derived in lines $2-3$ of the proof of Lemma 2.1 and use inequality (3.1) to obtain

$$
u\left(\mathfrak{A}_{n}\right) \Phi_{3}^{-1}\left(\frac{1}{\mu\left(\mathfrak{A}_{n}\right)}\right) \leq u\left(\mathfrak{A}_{n}\right) \frac{2 \Phi_{1}^{-1}\left(1 / \mu\left(\mathfrak{A}_{n}\right)\right)}{\Phi_{2}^{-1}\left(1 / \mu\left(\mathfrak{A}_{n}\right)\right)} \leq 2\left\|M_{u} f_{n}\right\|_{\Phi_{2}} \leq 2\left\|M_{u}\right\|<\infty
$$

This completes the proof. 
We have a straightforward consequence.

Corollary 3.5 Under the assumptions of Theorem 3.4, if $(\Omega, \Sigma, \mu)$ is a non-atomic measure space, then the multiplication operator $M_{u}$ is bounded from $L^{\Phi_{1}}(\Omega)$ into $L^{\Phi_{2}}(\Omega)$ if and only if $M_{u}=0$.

Now we present some sufficient conditions for the continuity of the operator $M_{u}$ from one Orlicz space into another.

Theorem 3.6 Let $\Phi_{1}, \Phi_{2}$ be Young functions vanishing only at zero, taking only finite values, and such that $\Phi_{1}, \Phi_{2} \in \Delta^{\prime}$ and $\Phi_{2} \circ \Phi_{1}^{-1}$ is a Young function. Then $u \in L_{+}^{0}(\Omega)$ induces a bounded multiplication operator $M_{u}: L^{\Phi_{1}}(\Omega) \rightarrow L^{\Phi_{2}}(\Omega)$ if

(i) $u(t)=0$ for $\mu$-a.e. $t \in \mathfrak{B}$;

(ii) $\sup _{n \in \mathbb{N}} \Phi_{2}\left[\frac{u\left(\mathfrak{A}_{n}\right)}{\Phi_{1}^{-1}\left(\mu\left(\mathfrak{A}_{n}\right)\right)}\right] \mu\left(\mathfrak{A}_{n}\right)<\infty$.

Proof Suppose that (i) and (ii) hold and set $M:=\sup _{n \in \mathbb{N}} \Phi_{2}\left[\frac{u\left(\mathfrak{A}_{n}\right)}{\Phi_{1}^{-1}\left(\mu\left(\mathfrak{A}_{n}\right)\right)}\right] \mu\left(\mathfrak{A}_{n}\right)$. Then for each $f \in L^{\Phi_{1}}(\Omega)$ we have

$$
\begin{aligned}
I_{\Phi_{2}}\left(M_{u} f\right) & =\int_{\Omega} \Phi_{2}\left(M_{u} f\right) d \mu=\int_{\mathfrak{B}} \Phi_{2}(u(t) f(t)) d \mu+\int_{\bigcup_{n} \mathfrak{A}_{n}} \Phi_{2}(u(t) f(t)) d \mu \\
& =\sum_{n \in \mathbb{N}} \int_{\mathfrak{A}_{n}} \Phi_{2}(u(t) f(t)) d \mu=\sum_{n \in \mathbb{N}} \Phi_{2}\left(u\left(\mathfrak{A}_{n}\right) f\left(\mathfrak{A}_{n}\right)\right) \mu\left(\mathfrak{A}_{n}\right) \\
& =\sum_{n \in \mathbb{N}} \Phi_{2}\left[\Phi_{1}^{-1}\left(\mu\left(\mathfrak{A}_{n}\right)\right) \Phi_{1}^{-1} \circ \Phi_{1}\left(f\left(\mathfrak{A}_{n}\right)\right) \frac{u\left(\mathfrak{A}_{n}\right)}{\Phi_{1}^{-1}\left(\mu\left(\mathfrak{A}_{n}\right)\right)}\right] \mu\left(\mathfrak{A}_{n}\right) \\
& \leq \sum_{n \in \mathbb{N}} \Phi_{2}\left[\Phi_{1}^{-1}\left[c_{1} \mu\left(\mathfrak{A}_{n}\right) \Phi_{1}\left(f\left(\mathfrak{A}_{n}\right)\right)\right] \frac{u\left(\mathfrak{A}_{n}\right)}{\Phi_{1}^{-1}\left(\mu\left(\mathfrak{A}_{n}\right)\right)}\right] \mu\left(\mathfrak{A}_{n}\right) \\
& \leq c_{2} \sum_{n \in \mathbb{N}} \Phi_{2} \circ \Phi_{1}^{-1}\left[c_{1} \mu\left(\mathfrak{A}_{n}\right) \Phi_{1}\left(f\left(\mathfrak{A}_{n}\right)\right)\right] \Phi_{2}\left[\frac{u\left(\mathfrak{A}_{n}\right)}{\Phi_{1}^{-1}\left(\mu\left(\mathfrak{A}_{n}\right)\right)}\right] \mu\left(\mathfrak{A}_{n}\right) \\
& \leq c_{2} \sum_{n \in \mathbb{N}} \Phi_{2} \circ \Phi_{1}^{-1}\left[c_{1} \mu\left(\mathfrak{A}_{n}\right) \Phi_{1}\left(f\left(\mathfrak{A}_{n}\right)\right)\right] M \\
& \leq c_{2} M \Phi_{2} \circ \Phi_{1}^{-1}\left[c_{1} \sum_{n \in \mathbb{N}} \mu\left(\mathfrak{A}_{n}\right) \Phi_{1}\left(f\left(\mathfrak{A}_{n}\right)\right)\right]
\end{aligned}
$$

where $c_{1}, c_{2}$ are positive constants related to conditions $\Phi_{1} \in \Delta^{\prime}$ and $\Phi_{2} \in \Delta^{\prime}$, respectively, and the last inequality follows from the superadditivity of the convex function $\Phi_{2} \circ \Phi_{1}^{-1}$ on the interval $[0, \infty)$. Now let $\|f\|_{\Phi_{1}} \leq 1$. We get

$$
I_{\Phi_{2}}\left(M_{u} f\right) \leq c_{2} M \Phi_{2} \circ \Phi_{1}^{-1}\left(c_{1}\right)<\infty
$$

This implies that $\left\|M_{u} f\right\|_{\Phi_{2}} \leq \max \left(c_{2} M \Phi_{2} \circ \Phi_{1}^{-1}\left(c_{1}\right), 1\right)$, and so $M_{u}$ is bounded. 
Remark 3.7 The following results on the classical Lebesgue spaces, presented in [38] (see also [18]), are an immediate consequence of Theorem 3.6.

1. Taking $\Phi_{1}(x)=|x|^{p} / p$ and $\Phi_{2}(x)=|x|^{q} / q$, where $1<p<q<\infty$, by Theorems 3.4 and 3.6, we obtain that the multiplication operator $M_{u}$ induced by a function $u \in L_{+}^{0}(\Omega)$ is bounded from $L^{p}(\Omega)$ into $L^{q}(\Omega)$ if and only if the following conditions hold:

(i) $u(t)=0$ for $\mu$-almost all $t \in \mathfrak{B}$;

(ii) $\sup _{n \in \mathbb{N}} \frac{u\left(\mathfrak{A}_{n}\right)^{r}}{\mu\left(\mathfrak{A}_{n}\right)}<\infty$, where $q^{-1}+r^{-1}=p^{-1}$.

2. Similarly, taking $\Phi_{1}(x)=|x|^{p} / p$ and $\Phi_{2}(x)=|x|^{q} / q$, where $1<q<p<\infty$, by O'Niel's theorem (see the beginning of Sect. 3 ) combined with Proposition 3.2 we obtain that the multiplication operator $M_{u}$ induced by a function $u \in L_{+}^{0}(\Omega)$ is bounded from $L^{p}(\Omega)$ into $L^{q}(\Omega)$ if and only if $u \in L^{r}(\Omega)$, where $p^{-1}+r^{-1}=$ $q^{-1}$.

\section{Boundedness of the composition operator}

In this section, we give necessary conditions and sufficient conditions under which the composition operator $C_{T}$ acts continuously between distinct Orlicz spaces.

Theorem 4.1 Let $T: \Omega \rightarrow \Omega$ be a non-singular measurable transformation and let $\Phi_{1}, \Phi_{2}, \Phi_{3}$ be Young functions vanishing only at zero, taking only finite values, and such that $\Phi_{1}(x y) \leq \Phi_{2}(x)+\Phi_{3}(y)$ for all $x, y \geq 0$. If $T$ induces a bounded composition operator $C_{T}: L^{\Phi_{1}}(\Omega) \rightarrow L^{\Phi_{2}}(\Omega)$, then $h(t)=0$ for $\mu$-almost all $t \in T(B)$.

Proof Suppose, towards a contradiction, that $\mu(\{t \in T(\mathfrak{B}): h(t) \neq 0\})>0$. Then there is a constant $\delta>0$ such that the set $E=\{t \in T(\mathfrak{B}): h(t)>\delta\}$ has positive measure. Since $\mu(E)>0, E$ is non-atomic, and $\Phi_{1}(x y) \leq \Phi_{2}(x)+\Phi_{3}(y)$ for all $x, y \geq 0$, by Lemmas 2.1 and 2.3 we have that there exists $f \in L^{\Phi_{1}}(\Omega)$ such that $f_{\left.\right|_{E}} \notin L^{\Phi_{2}}(E)$. Since $E \subset T(\mathfrak{B})$, we obtain that $C_{T} f \neq 0$ (otherwise we would have $\left.f_{\left.\right|_{E}} \in L^{\Phi_{2}}(E)\right)$. Together with the fact that $C_{T}$ is bounded, the above gives

$$
\begin{aligned}
\infty=\int_{E} \delta \Phi_{2}\left(\frac{f(t)}{\left\|C_{T} f\right\|_{\Phi_{2}}}\right) d \mu & \leq \int_{E} h(t) \Phi_{2}\left(\frac{f(t)}{\left\|C_{T} f\right\|_{\Phi_{2}}}\right) d \mu \\
& \leq \int_{\Omega} \Phi_{2}\left(\frac{C_{T} f(t)}{\left\|C_{T} f\right\|_{\Phi_{2}}}\right) d \mu \leq 1
\end{aligned}
$$

which is a contradiction finishing the proof.

Remark 4.2 Although the function $\Phi_{3}$ does not feature in the thesis of Theorem 4.1, it was actually used in the proof via Lemma 2.1.

Theorem 4.3 Let $T: \Omega \rightarrow \Omega$ be a non-singular measurable transformation, and let $\Phi_{1}, \Phi_{2}$ be Young functions vanishing only at zero, taking only finite values, and such that $\Phi_{1}, \Phi_{2} \in \Delta^{\prime}$ and $\Phi_{2} \circ \Phi_{1}^{-1}$ is a Young function. Then $T$ induces a bounded composition operator $C_{T}: L^{\Phi_{1}}(\Omega) \rightarrow L^{\Phi_{2}}(\Omega)$ if 
(i) $h(t)=0$ for $\mu$-almost all $t \in T(\mathfrak{B})$;

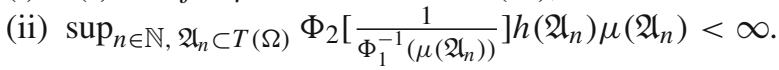

Proof Assume that (i) and (ii) hold and put

$$
M:=\sup _{n \in \mathbb{N}, \mathfrak{A}_{n} \subset T(\Omega)} \Phi_{2}\left[\frac{1}{\Phi_{1}^{-1}\left(\mu\left(\mathfrak{A}_{n}\right)\right)}\right] h\left(\mathfrak{A}_{n}\right) \mu\left(\mathfrak{A}_{n}\right) .
$$

Then for each $f \in L^{\Phi_{1}}(\Omega)$ we have

$$
\begin{aligned}
I_{\Phi_{2}}\left(C_{T} f\right) & =\int_{\Omega} \Phi_{2}\left(C_{T} f\right) d \mu=\int_{T(\mathfrak{B})} h(t) \Phi_{2}(f(t)) d \mu+\int_{\bigcup_{n} T\left(\mathfrak{A}_{n}\right)} h(t) \Phi_{2}(f(t)) d \mu \\
& =\sum_{\mathfrak{A}_{n} \subset T(\Omega)} \int_{\mathfrak{A}_{n}} h(t) \Phi_{2}(f(t)) d \mu=\sum_{\mathfrak{A}_{n} \subset T(\Omega)} h\left(\mathfrak{A}_{n}\right) \Phi_{2}\left(f\left(\mathfrak{A}_{n}\right)\right) \mu\left(\mathfrak{A}_{n}\right) \\
& =\sum_{\mathfrak{A}_{n} \subset T(\Omega)} h\left(\mathfrak{A}_{n}\right) \Phi_{2}\left[\Phi_{1}^{-1}\left(\mu\left(\mathfrak{A}_{n}\right)\right) \Phi_{1}^{-1} \circ \Phi_{1}\left(f\left(\mathfrak{A}_{n}\right)\right) \frac{1}{\Phi_{1}^{-1}\left(\mu\left(\mathfrak{A}_{n}\right)\right)}\right] \mu\left(\mathfrak{A}_{n}\right) \\
& \leq \sum_{\mathfrak{A}_{n} \subset T(\Omega)} h\left(\mathfrak{A}_{n}\right) \Phi_{2}\left[\Phi_{1}^{-1}\left[c_{1} \mu\left(\mathfrak{A}_{n}\right) \Phi_{1}\left(f\left(\mathfrak{A}_{n}\right)\right)\right] \frac{1}{\Phi_{1}^{-1}\left(\mu\left(\mathfrak{A}_{n}\right)\right)}\right] \mu\left(\mathfrak{A}_{n}\right) \\
& \leq c_{2} \sum_{\mathfrak{A}_{n} \subset T(\Omega)} h\left(\mathfrak{A}_{n}\right) \Phi_{2} \circ \Phi_{1}^{-1}\left[c_{1} \mu\left(\mathfrak{A}_{n}\right) \Phi_{1}\left(f\left(\mathfrak{A}_{n}\right)\right)\right] \Phi_{2}\left[\frac{1}{\Phi_{1}^{-1}\left(\mu\left(\mathfrak{A}_{n}\right)\right)}\right] \mu\left(\mathfrak{A}_{n}\right) \\
& \leq c_{2} \sum_{\mathfrak{A}_{n} \subset T(\Omega)} \Phi_{2} \circ \Phi_{1}^{-1}\left[c_{1} \mu\left(\mathfrak{A}_{n}\right) \Phi_{1}\left(f\left(\mathfrak{A}_{n}\right)\right)\right] M \\
& \leq c_{2} M \Phi_{2} \circ \Phi_{1}^{-1}\left[c_{1} \sum_{n \in \mathbb{N}} \mu\left(\mathfrak{A}_{n}\right) \Phi_{1}\left(f\left(\mathfrak{A}_{n}\right)\right)\right],
\end{aligned}
$$

where $c_{1}, c_{2}$ are positive constants related to conditions $\Phi_{1} \in \Delta^{\prime}$ and $\Phi_{2} \in \Delta^{\prime}$, respectively, and the last inequality follows from the superadditivity of the convex function $\Phi_{2} \circ \Phi_{1}^{-1}$ on the interval $[0, \infty)$. Now let $\|f\|_{\Phi_{1}} \leq 1$. We obtain

$$
I_{\Phi_{2}}\left(C_{T} f\right) \leq c_{2} M \Phi_{2} \circ \Phi_{1}^{-1}\left(c_{1}\right)<\infty
$$

Hence $\left\|C_{T} f\right\|_{\Phi_{2}} \leq \max \left(c_{2} M \Phi_{2} \circ \Phi_{1}^{-1}\left(c_{1}\right), 1\right)$, and so $C_{T}$ is bounded.

Theorem 4.4 Let $T: \Omega \rightarrow \Omega$ be a non-singular measurable transformation, and let $\Phi_{1}, \Phi_{2}, \Phi_{3}$ be Young functions vanishing only at zero, taking only finite values, and such that $\Phi_{1}(x y) \leq \Phi_{2}(x)+\Phi_{3}(y)$ for all $x, y \geq 0$. Consider the following statements:

(i) $T$ induces a bounded composition operator $C_{T}: L^{\Phi_{1}}(\Omega) \rightarrow L^{\Phi_{2}}(\Omega)$.

(ii) $\mu(\mathfrak{B})=0$ and there is a constant $M>0$ such that $\Phi_{1}^{-1}\left(\frac{1}{\mu\left(\mathfrak{A}_{n}\right)}\right) \leq$ $M \Phi_{2}^{-1}\left(\frac{1}{h\left(\mathfrak{A}_{n}\right) \mu\left(\mathfrak{A}_{n}\right)}\right)$ for all $n \in \mathbb{N}$ such that $\mathfrak{A}_{n} \subset T(\Omega)$. 
(iii) $h(t)=0$ for $\mu$-a.e. $t \in T(\mathfrak{B})$ and

$$
\sup _{n \in \mathbb{N}, \mathfrak{A}_{n} \subset T(\Omega)} \frac{\Phi_{2}^{-1}\left(\frac{1}{\mu\left(\mathfrak{A}_{n}\right)}\right) \Phi_{3}^{-1}\left(\frac{1}{\mu\left(\mathfrak{A}_{n}\right)}\right)}{\Phi_{2}^{-1}\left(\frac{1}{h\left(\mathfrak{A}_{n}\right) \mu\left(\mathfrak{A}_{n}\right)}\right)}<\infty .
$$

(iv) $h(t)=0$ for $\mu$-a.e. $t \in T(\mathfrak{B})$ and

$$
\sup _{n \in \mathbb{N}, \mathfrak{A}_{n} \subset T(\Omega)} \Phi_{2}^{-1}\left(h\left(\mathfrak{A}_{n}\right)\right) \Phi_{3}^{-1}\left(\frac{1}{\mu\left(\mathfrak{A}_{n}\right)}\right)<\infty .
$$

Then $(i) \Rightarrow(i i) \Rightarrow\left(\right.$ iii). Moreover, if $\Phi_{2} \in \Delta^{\prime}$, then (ii) $\Rightarrow$ (iv).

Proof To prove that (i) $\Rightarrow$ (ii), assume that $C_{T}$ is a bounded operator from $L^{\Phi_{1}}(\Omega)$ into $L^{\Phi_{2}}(\Omega)$. It implies that there is a constant $M>0$ such that for every $n \in \mathbb{N}$,

$$
\left\|C_{T} \chi_{\mathfrak{A}_{n}}\right\|_{\Phi_{2}} \leq M\left\|\chi_{\mathfrak{A}_{n}}\right\|_{\Phi_{1}},
$$

where $\chi_{\mathfrak{A}_{n}}$ is the characteristic function of the atom $\mathfrak{A}_{n} \subset T(\Omega)$. Calculating the norm of the characteristic function $\chi_{\mathfrak{A}_{n}}$, we obtain

$$
\frac{1}{\Phi_{2}^{-1}\left(\frac{1}{\mu \circ T^{-1}\left(\mathfrak{A}_{n}\right)}\right)} \leq \frac{M}{\Phi_{1}^{-1}\left(\frac{1}{\mu\left(\mathfrak{A}_{n}\right)}\right)}
$$

which yields the desired inequality.

By Theorem 4.1, we have $h(t)=0$ for $\mu$-a.e. $t \in T(\mathfrak{B})$, hence $\mu(\mathfrak{B})=\mu \circ$ $T^{-1}(T(\mathfrak{B}))=\int_{T(\mathfrak{B})} h(t) d \mu(t)=0$.

Next we show that (ii) $\Rightarrow$ (iii). For each $\mathfrak{A}_{n} \subset T(\Omega)$ let

$$
\Phi_{1}^{-1}\left(\frac{1}{\mu\left(\mathfrak{A}_{n}\right)}\right) \leq M \Phi_{2}^{-1}\left(\frac{1}{h\left(\mathfrak{A}_{n}\right) \mu\left(\mathfrak{A}_{n}\right)}\right) .
$$

Since $\Phi_{1}(x y) \leq \Phi_{2}(x)+\Phi_{3}(y)$ for all $x, y \geq 0$, we have $\Phi_{3}^{-1}(x) \Phi_{2}^{-1}(x) \leq 2 \Phi_{1}^{-1}(x)$ for all $x \geq 0$. Plugging $x=\frac{1}{\mu\left(\mathfrak{A}_{n}\right)}$ in this inequality and using (4.1), we have

$$
\begin{aligned}
\Phi_{3}^{-1}\left(\frac{1}{\mu\left(\mathfrak{A}_{n}\right)}\right) \Phi_{2}^{-1}\left(\frac{1}{\mu\left(\mathfrak{A}_{n}\right)}\right) & \leq 2 \Phi_{1}^{-1}\left(\frac{1}{\mu\left(\mathfrak{A}_{n}\right)}\right) \\
& \leq 2 M \Phi_{2}^{-1}\left(\frac{1}{h\left(\mathfrak{A}_{n}\right) \mu\left(\mathfrak{A}_{n}\right)}\right)
\end{aligned}
$$

Hence

$$
\sup _{n \in \mathbb{N}, \mathfrak{A}_{n} \subset T(\Omega)} \frac{\Phi_{2}^{-1}\left(\frac{1}{\mu\left(\mathfrak{A}_{n}\right)}\right) \Phi_{3}^{-1}\left(\frac{1}{\mu\left(\mathfrak{A}_{n}\right)}\right)}{\Phi_{2}^{-1}\left(\frac{1}{h\left(\mathfrak{A}_{n}\right) \mu\left(\mathfrak{A}_{n}\right)}\right)} \leq 2 M<\infty .
$$


Moreover, since $\mu \circ T^{-1}(T(\mathfrak{B}))=\mu(\mathfrak{B})=0$, we have $h(t)=0$ for $\mu$-a.e. $t \in T(\mathfrak{B})$.

Finally, we prove implication (ii) $\Rightarrow$ (iv) under the assumption that $\Phi_{2} \in \Delta^{\prime}$, i.e. that there is a constant $c>0$ such that $\Phi_{2}^{-1}(x) \Phi_{2}^{-1}(y) \leq \Phi_{2}^{-1}(c x y)(x, y \geq 0)$. We may assume that $c \geq 1$. Plugging $x=1 / h\left(\mathfrak{A}_{n}\right) \mu\left(\mathfrak{A}_{n}\right)$ and $y=h\left(\mathfrak{A}_{n}\right) / c$ in this inequality, we get

$$
\Phi_{2}^{-1}\left(\frac{1}{h\left(\mathfrak{A}_{n}\right) \mu\left(\mathfrak{A}_{n}\right)}\right) \leq \frac{\Phi_{2}^{-1}\left(\frac{1}{\mu\left(\mathfrak{A}_{n}\right)}\right)}{\Phi_{2}^{-1}\left(\frac{h\left(\mathfrak{A}_{n}\right)}{c}\right)} .
$$

Combining inequality (4.2) with this, yields

$$
\Phi_{3}^{-1}\left(\frac{1}{\mu\left(\mathfrak{A}_{n}\right)}\right) \Phi_{2}^{-1}\left(\frac{1}{\mu\left(\mathfrak{A}_{n}\right)}\right) \leq 2 M \Phi_{2}^{-1}\left(\frac{1}{h\left(\mathfrak{A}_{n}\right) \mu\left(\mathfrak{A}_{n}\right)}\right) \leq 2 M \frac{\Phi_{2}^{-1}\left(\frac{1}{\mu\left(\mathfrak{A}_{n}\right)}\right)}{\Phi_{2}^{-1}\left(\frac{h\left(\mathfrak{A}_{n}\right)}{c}\right)} .
$$

Therefore

$$
\Phi_{2}^{-1}\left(\frac{h\left(\mathfrak{A}_{n}\right)}{c}\right) \Phi_{3}^{-1}\left(\frac{1}{\mu\left(\mathfrak{A}_{n}\right)}\right) \leq 2 M
$$

Since $\Phi_{2}^{-1}$ is a concave function and $c \geq 1$, we have

$$
\frac{1}{c} \Phi_{2}^{-1}\left(h\left(\mathfrak{A}_{n}\right)\right) \Phi_{3}^{-1}\left(\frac{1}{\mu\left(\mathfrak{A}_{n}\right)}\right) \leq 2 M,
$$

hence $\sup _{n \in \mathbb{N}, \mathfrak{A}_{n} \subset T(\Omega)} \Phi_{2}^{-1}\left(h\left(\mathfrak{A}_{n}\right)\right) \Phi_{3}^{-1}\left(1 / \mu\left(\mathfrak{A}_{n}\right)\right) \leq 2 M c<\infty$, and this completes the proof.

Now we present two lemmas on the relationship between multiplication operators and composition operators, which will be needed later on.

Lemma 4.5 Let $T: \Omega \rightarrow \Omega$ be a non-singular measurable transformation, and let $\Phi_{1}, \Phi_{2}$ be Young functions vanishing only at zero and taking only finite values, with $\Phi_{2} \in \nabla^{\prime}$. For any function $f \in L^{\Phi_{1}}(\Omega)$ such that $M_{\Phi_{2}^{-1} \circ h} f \in L^{\Phi_{2}}(T(\Omega))$, we have $C_{T} f \in L^{\Phi_{2}}(\Omega)$ and the following inequality holds

$$
\left\|C_{T} f\right\|_{\Phi_{2}} \leq b\left\|M_{\Phi_{2}^{-1} \circ h} f\right\|_{\Phi_{2}},
$$

where $b$ is some positive constant.

Proof Let $f \in L^{\Phi_{1}}(\Omega)$ be such that $M_{\Phi_{2}^{-1} \circ h} f \in L^{\Phi_{2}}(T(\Omega))$. By the definition of the norm $\|\cdot\|_{\Phi_{2}}$ and the assumption that $\Phi_{2} \in \nabla^{\prime}$, for $b>0$ such that $\Phi_{2}(b x y) \geq$ $\Phi_{2}(x) \Phi_{2}(y) \quad(x, y \geq 0)$ we have

$$
\left\|C_{T} f\right\|_{\Phi_{2}}=\inf \left\{k: \int_{\Omega} \Phi_{2}\left(\frac{f(T(t))}{k}\right) d \mu \leq 1\right\}
$$




$$
\begin{aligned}
& =\inf \left\{k: \int_{T(\Omega)} h(t) \Phi_{2}\left(\frac{f(t)}{k}\right) d \mu \leq 1\right\} \\
& =\inf \left\{k: \int_{T(\Omega)} \Phi_{2}\left(\Phi_{2}^{-1}(h(t)) \Phi_{2}\left(\frac{f(t)}{k}\right) d \mu \leq 1\right\}\right. \\
& \leq \inf \left\{k: \int_{T(\Omega)} \Phi_{2}\left(\frac{b \Phi_{2}^{-1}(h(t)) f(t)}{k}\right) d \mu \leq 1\right\} \\
& =b \inf \left\{k / b: \int_{T(\Omega)} \Phi_{2}\left(\frac{\Phi_{2}^{-1}(h(t)) f(t)}{k / b}\right) d \mu \leq 1\right\} \\
& =b\left\|M_{\Phi_{2}^{-1} \circ h} f\right\|_{\Phi_{2} .}
\end{aligned}
$$

Corollary 4.6 Under the assumptions of Lemma 4.5, if $M_{\Phi_{2}^{-1} \circ h}$ is a bounded multiplication operator from $L^{\Phi_{1}}(T(\Omega))$ into $L^{\Phi_{2}}(T(\Omega))$, then $C_{T}$ is a bounded composition operator from $L^{\Phi_{1}}(\Omega)$ into $L^{\Phi_{2}}(\Omega)$.

Lemma 4.7 Let $T: \Omega \rightarrow \Omega$ be a non-singular measurable transformation and let $\Phi_{1}, \Phi_{2}$ be Young functions vanishing only at zero and taking only finite values, with $\Phi_{2} \in \Delta^{\prime}$. For any function $f \in L^{\Phi_{1}}(\Omega)$ such that $C_{T} f \in L^{\Phi_{2}}(\Omega)$, we have $M_{\Phi_{2}^{-1} \circ h} f \in L^{\Phi_{2}}(T(\Omega))$ and the following inequality holds

$$
\left\|M_{\Phi_{2}^{-1} \circ h} f\right\|_{\Phi_{2}} \leq c\left\|C_{T} f\right\|_{\Phi_{2}}
$$

where c is some positive constant.

Proof Let $f \in L^{\Phi_{1}}(\Omega)$ be such that $C_{T} f \in L^{\Phi_{2}}(\Omega)$, and let $c \geq 1$ be a constant in the inequality $\Phi_{2}(x y) \leq c \Phi_{2}(x) \Phi_{2}(y)$ for all $x, y \geq 0$. Then

$$
\begin{aligned}
\left\|M_{\Phi_{2}^{-1} \circ h} f\right\|_{\Phi_{2}} & =\inf \left\{k: \int_{T(\Omega)} \Phi_{2}\left(\frac{\Phi_{2}^{-1}(h(t)) f(t)}{k}\right) d \mu \leq 1\right\} \\
& \leq \inf \left\{k: \int_{T(\Omega)} c \Phi_{2}\left(\Phi_{2}^{-1}(h(t))\right) \Phi_{2}\left(\frac{f(t)}{k}\right) d \mu \leq 1\right\} \\
& =\inf \left\{k: \int_{T(\Omega)} c h(t) \Phi_{2}\left(\frac{f(t)}{k}\right) d \mu \leq 1\right\} \\
& =\inf \left\{k: \int_{\Omega} c \Phi_{2}\left(\frac{f(T(t))}{k}\right) d \mu \leq 1\right\} \\
& \leq \inf \left\{k: \int_{\Omega} \Phi_{2}\left(\frac{f(T(t))}{k / c}\right) d \mu \leq 1\right\} \\
& =c \inf \left\{k / c: \int_{\Omega} \Phi_{2}\left(\frac{f(T(t))}{k / c}\right) d \mu \leq 1\right\} \\
& =c\left\|C_{T} f\right\|_{\Phi_{2}},
\end{aligned}
$$


as required.

Corollary 4.8 Under the assumptions of Lemma 4.7, if $C_{T}$ is a bounded composition operator from $L^{\Phi_{1}}(\Omega)$ into $L^{\Phi_{2}}(\Omega)$, then $M_{\Phi_{2}^{-1} \circ h}$ is a bounded multiplication operator from $L^{\Phi_{1}}(T(\Omega))$ into $L^{\Phi_{2}}(T(\Omega))$.

Following [38], for any $F$ in the $\sigma$-algebra $\Sigma \cap T(\Omega)$, we define

$$
Q_{T}(F):=\inf \left\{b \geq 0: \mu \circ T^{-1}(E) \leq b \mu(E), E \in \Sigma \cap T(\Omega), E \subset F\right\}
$$

To prove our next results, we will need the following two lemmas.

Lemma 4.9 [38, Lemma 3.6] For any $F \in \Sigma \cap T(\Omega)$, we have $Q_{T}(F)=$ $\operatorname{ess}_{\sup _{t \in F}} h(t)$, where $h=d\left(\mu \circ T^{-1}\right) / d \mu$.

Lemma 4.10 Let $T$ be a measurable transformation of the measure space $(\Omega, \Sigma, \mu)$ into itself and let $\Phi$ be a Young function. Then

$$
\int_{T(\Omega)} \Phi \circ h d \mu=\inf \left\{\sum_{j=1}^{\infty} \Phi\left(Q_{T}\left(F_{j}\right)\right) \mu\left(F_{j}\right):\left\{F_{j}\right\} \in \mathcal{P}_{T(\Omega)}\right\}
$$

where $\mathcal{P}_{T(\Omega)}$ is the family of all measurable partitions of $T(\Omega)$ and the case $\infty=\infty$ is admissible.

Proof Let $I:=\inf \left\{\sum_{j=1}^{\infty} \Phi\left(Q_{T}\left(F_{j}\right)\right) \mu\left(F_{j}\right):\left\{F_{j}\right\} \in \mathcal{P}_{T(\Omega)}\right\}$. By Lemma 4.9, for the partition $\left\{F_{j}\right\}$ of $T(\Omega)$, we have

$$
\begin{aligned}
\int_{T(\Omega)} \Phi \circ h d \mu & =\sum_{j=1}^{\infty} \int_{F_{j}} \Phi(h(t)) d \mu \leq \sum_{j=1}^{\infty} \Phi\left(\operatorname{ess~sup}_{t \in F_{j}} h(t)\right) \mu\left(F_{j}\right) \\
& =\sum_{j=1}^{\infty} \Phi\left(Q_{T}\left(F_{j}\right)\right) \mu\left(F_{j}\right) .
\end{aligned}
$$

Hence

$$
\int_{T(\Omega)} \Phi \circ h d \mu \leq I
$$

To prove the reverse inequality, let $a>1$ be an arbitrary number. For each $m \in \mathbb{Z}$ define the set

$$
G_{m}:=\left\{t \in T(\Omega): a^{m-1} \leq \Phi(h(t))<a^{m}\right\} .
$$

Let $\left\{F_{j}\right\}_{j=1}^{\infty}$ be any rearrangement of the sets $\left\{G_{m}\right\}_{m=-\infty}^{\infty}$ and the set $\{t \in T(\Omega)$ : $h(t)=0\}$. Clearly, $\left\{F_{j}\right\}_{j=1}^{\infty}$ is a partition of $T(\Omega)$. By Lemma 4.9, we have 


$$
\begin{aligned}
I & \leq \sum_{j=1}^{\infty} \Phi\left(Q_{T}\left(F_{j}\right)\right) \mu\left(F_{j}\right)=\sum_{j=1}^{\infty} \Phi\left(\operatorname{ess~sup}_{t \in F_{j}} h(t)\right) \mu\left(F_{j}\right) \\
& =\sum_{m=-\infty}^{\infty} \Phi\left(\operatorname{ess} \sup _{t \in G_{m}} h(t)\right) \mu\left(G_{m}\right) \leq \sum_{m=-\infty}^{\infty} a^{m} \mu\left(G_{m}\right) \\
& =a \sum_{m=-\infty}^{\infty} a^{m-1} \mu\left(G_{m}\right)=a \sum_{m=-\infty}^{\infty} \int_{G_{m}} a^{m-1} d \mu \\
& \leq a \sum_{m=-\infty}^{\infty} \int_{G_{m}} \Phi \circ h d \mu=a \sum_{j=1}^{\infty} \int_{F_{j}} \Phi \circ h d \mu \\
& =a \int_{T(\Omega)} \Phi \circ h d \mu .
\end{aligned}
$$

Since this holds for any $a>1$, the result follows.

Proposition 3.2 and Lemma 4.7 allow us to give a necessary condition for the boundedness of the composition operator $C_{T}$. Statement (ii) of the next theorem is interesting, because under some assumptions on the Young functions $\Phi_{1}, \Phi_{2}$ it gives the information that if $C_{T}$ acts continuously from $L^{\Phi_{1}}(\Omega)$ into $L^{\Phi_{2}}(\Omega)$, then the Radon-Nikodym derivative $h$ must be from a concrete Orlicz space $L^{\Phi}(\Omega)$, where $\Phi$ depends on $\Phi_{1}$ and $\Phi_{2}$.

Theorem 4.11 Let $T$ be a non-singular measurable transformation of the measure space $(\Omega, \Sigma, \mu)$ into itself. Then the following assertions hold.

(i) If $\Phi$ is a Young function, then the Radon-Nikodym derivative $h=d\left(\mu \circ T^{-1}\right) / d \mu$ belongs to $L^{\Phi}(T(\Omega))$ if and only if there exists a partition $\left\{F_{j}\right\}_{j=1}^{\infty}$ of $T(\Omega)$ and $\lambda>0$ such that $\sum_{j=1}^{\infty} \Phi\left(\lambda Q_{T}\left(F_{j}\right)\right) \mu\left(F_{j}\right)<\infty$.

(ii) Let $\Phi_{1}$ be an N-function, $\Phi_{2}$ a Young function vanishing only at zero and taking only finite values, such that $\Phi_{1} \in \Delta^{\prime}, \Phi_{2} \in \Delta_{2}$, and $\Phi_{3}:=\Psi_{2} \circ \Psi_{1}^{-1}$ is a Young function. If $T$ induces a bounded composition operator $C_{T}: L^{\Phi_{1}}(\Omega) \rightarrow$ $L^{\Phi_{2}}(\Omega)$, then the Radon-Nikodym derivative $h=d\left(\mu \circ T^{-1}\right) / d \mu$ belongs to $L^{\Psi_{3} \circ \Psi_{1} \circ \Phi_{2}^{-1}}(T(\Omega))$.

Proof (i) It is an easy consequence of Lemma 4.10 with the function $\Phi_{\lambda}(x):=\Phi(\lambda x)$ in place of the Young function $\Phi$.

(ii) Since, by assumption, $C_{T}$ is a bounded composition operator from $L^{\Phi_{1}}(\Omega)$ into $L^{\Phi_{2}}(\Omega)$, Lemma 4.7 implies that

$$
M_{\Phi_{2}^{-1} \circ h}: L^{\Phi_{1}}(T(\Omega)) \rightarrow L^{\Phi_{2}}(T(\Omega))
$$

is a bounded multiplication operator. Thus, by Proposition 3.2, for the Young function $\Phi_{3}=\Psi_{2} \circ \Psi_{1}^{-1}$, we have $\Phi_{2}^{-1} \circ h \in L^{\Psi_{3} \circ \Psi_{1}}(T(\Omega))$, and so

$$
\int_{T(\Omega)} \Psi_{3} \circ \Psi_{1} \circ \lambda \Phi_{2}^{-1} \circ h d \mu<\infty
$$


for some constant $\lambda>0$. Since $\Psi_{3} \circ \Psi_{1}$ is a nondecreasing function on the interval $[0, \infty)$, we may assume that $0<\lambda<1$. It can be easily shown that the $\Delta_{2}$-condition assumed for the function $\Phi_{2}$ is equivalent to the condition: for all $\lambda \in(0,1)$ there is a constant $K>0$ such that $\Phi_{2}^{-1}(K x) \leq \lambda \Phi_{2}^{-1}(x)(x \geq 0)$. Since $\Psi_{3} \circ \Psi_{1}$ is a nondecreasing function on the interval $[0, \infty)$, we then get

$$
\int_{T(\Omega)} \Psi_{3} \circ \Psi_{1} \circ \Phi_{2}^{-1} \circ K h d \mu<\infty .
$$

Thus $h \in L^{\Psi_{3} \circ \Psi_{1} \circ \Phi_{2}^{-1}}(T(\Omega))$.

Finally, we give a sufficient condition for the composition operator $C_{T}$ to be bounded from $L^{\Phi_{1}}(\Omega)$ into $L^{\Phi_{2}}(\Omega)$ when $\Phi_{1} \circ \Phi_{2}^{-1}$ is a Young function.

Theorem 4.12 Let $\Phi_{1}, \Phi_{2}$ be Young functions vanishing only at zero and taking only finite values. If $\Phi_{3}=\Phi_{1} \circ \Phi_{2}^{-1}$ is a Young function and $h \in L^{\Psi_{3}}(T(\Omega))$, then $T$ induces a bounded composition operator $C_{T}: L^{\Phi_{1}}(\Omega) \rightarrow L^{\Phi_{2}}(\Omega)$.

Proof Since

$$
\begin{aligned}
I_{\Phi_{3}}\left(\Phi_{2} \circ f\right)=\int_{\Omega} \Phi_{3} \circ \Phi_{2} \circ f d \mu & =\int_{\Omega} \Phi_{1} \circ \Phi_{2}^{-1} \circ \Phi_{2} \circ f d \mu \\
& =\int_{\Omega} \Phi_{1} \circ f d \mu=I_{\Phi_{1}}(f),
\end{aligned}
$$

we have $f \in L^{\Phi_{1}}(\Omega)$ if and only if $\Phi_{2} \circ f \in L^{\Phi_{3}}(\Omega)$, and furthermore that $\| \Phi_{2} \circ$ $f\left\|_{\Phi_{3}}=\right\| f \|_{\Phi_{1}}$. Hence an application of Hölder's inequality yields

$$
\begin{aligned}
I_{\Phi_{2}}\left(C_{T} f\right) & =\int_{\Omega} \Phi_{2} \circ f \circ T d \mu=\int_{T(\Omega)} h \Phi_{2} \circ f d \mu \\
& \leq 2\|h\|_{\Psi_{3}}\left\|\Phi_{2} \circ f\right\|_{\Phi_{3}}=2\|h\|_{\Psi_{3}}\|f\|_{\Phi_{1}} .
\end{aligned}
$$

Thus $\left\|C_{T}\right\| \leq \max \left(2\|h\|_{\Psi_{3}}, 1\right)$, and this completes the proof.

Remark 4.13 We conclude this section with some applications of the theorems proved.

1. Taking $\Phi_{1}(x)=|x|^{p} / p$ and $\Phi_{2}(x)=|x|^{q} / q$, where $1<p<q<\infty$, by Theorems 4.1 and 4.4, we obtain that if $C_{T}$ is a composition operator induced by the non-singular measurable transformation $T: \Omega \rightarrow \Omega$, then the following statements are equivalent:

(a) $C_{T}$ is bounded from $L^{p}(\Omega)$ into $L^{q}(\Omega)$.

(b) $h(t)=0$ for $\mu$-a.e. $t \in T(\mathfrak{B})$ and $\sup _{n \in \mathbb{N}, \mathfrak{A}_{n} \subset T(\Omega)} \frac{h\left(\mathfrak{A}_{n}\right)^{p}}{\mu\left(\mathfrak{A}_{n}\right)^{q-p}}<\infty$.

(c) $\mu(\mathfrak{B})=0$ and there is a constant $k>0$ such that $\mu \circ T^{-1}\left(\mathfrak{A}_{n}\right)^{p} \leq k \mu\left(\mathfrak{A}_{n}\right)^{q}$ for all $n \in \mathbb{N}, \mathfrak{A}_{n} \subset T(\Omega)$.

2. Similarly, taking $\Phi_{1}(x)=|x|^{p} / p$ and $\Phi_{2}(x)=|x|^{q} / q$, where $1<q<p<\infty$, by Theorems 4.11 and 4.12, we obtain that if $C_{T}$ is a composition operator induced by the non-singular measurable transformation $T: \Omega \rightarrow \Omega$, then the following statements are equivalent: 
(a) $C_{T}$ is a bounded operator from $L^{p}(\Omega)$ into $L^{q}(\Omega)$.

(b) $h \in L^{\frac{r}{q}}(T(\Omega))$, where $p^{-1}+r^{-1}=q^{-1}$.

(c) There exists a partition $\left\{F_{j}\right\}_{j=1}^{\infty}$ of $T(\Omega)$ such that $\sum_{j=1}^{\infty} Q_{T}\left(F_{j}\right)^{\frac{r}{q}} \mu\left(F_{j}\right)<$ $\infty$.

These characterizations are due to Takagi and Yokouchi [38].

\section{Multiplication and composition operators with closed-range and/or finite rank}

In this section we are going to investigate closed-range multiplication and composition operators between distinct Orlicz spaces.

We start with a basic observation concerning Young functions.

Lemma 5.1 Let $\Phi_{1}, \Phi_{2}$ be Young functions such that $\Phi_{1}$ is an $N$-function and $\Phi_{3}:=$ $\Psi_{2} \circ \Psi_{1}^{-1}$ is a Young function. If $\Psi_{1} \in \Delta^{\prime}$, then there is a constant $b>0$ such that $b \Psi_{1}(x y) \leq \Psi_{2}(x)+\Psi_{3}\left(\Psi_{1}(y)\right)$ for all $x, y \geq 0$.

Proof By the definition of the function $\Phi_{3}$, we have

$$
\Psi_{3}(y)=\sup \left\{x|y|-\Phi_{3}(x): x \geq 0\right\}=\sup \left\{x|y|-\Psi_{2} \circ \Psi_{1}^{-1}(x): x \geq 0\right\} .
$$

Since $\Phi_{1}$ is an $N$-function, $\Psi_{1}$ is again an $N$-function, and $a_{\Psi_{1}}=0, b_{\Psi_{1}}=\infty$, So the usual inverse function $\Psi_{1}^{-1}$ exists. By substitution, we have

$$
\Psi_{3}\left(\Psi_{1}(y)\right)=\sup \left\{x \Psi_{1}(y)-\Psi_{2} \circ \Psi_{1}^{-1}(x): x \geq 0\right\},
$$

hence

$$
\Psi_{3}\left(\Psi_{1}(y)\right)=\sup \left\{\Psi_{1}(x) \Psi_{1}(y)-\Psi_{2}(x): x \geq 0\right\} .
$$

Since $\Psi_{1} \in \Delta^{\prime}$, i.e. $\Psi_{1}(x y) \leq c \Psi_{1}(x) \Psi_{1}(y)$ for some $c>0$ and all $x, y \geq 0$, we get from (5.1) that

$$
\Psi_{3}\left(\Psi_{1}(y)\right) \geq \frac{1}{c} \Psi_{1}(x y)-\Psi_{2}(x)
$$

for all $x, y \geq 0$, whence the desired inequality with $b=1 / c$ follows.

Now we characterize closed-range multiplication operators $M_{u}: L^{\Phi_{1}}(\Omega) \rightarrow$ $L^{\Phi_{2}}(\Omega)$ under the assumption $\Phi_{2}(x y) \leq \Phi_{1}(x)+\Phi_{3}(y)$ for all $x, y \geq 0$.

Theorem 5.2 Let $\Phi_{1}, \Phi_{2}, \Phi_{3}$, be Young functions vanishing only at zero, taking only finite values, and such that $\Phi_{2}, \Phi_{3} \in \Delta_{2}$ and $\Phi_{2}(x y) \leq \Phi_{1}(x)+\Phi_{3}(y)$ for all $x, y \geq 0$. If $u \in L_{+}^{\Phi_{3}}(\Omega)$, then $M_{u}$ is a bounded multiplication operator from $L^{\Phi_{1}}(\Omega)$ into $L^{\Phi_{2}}(\Omega)$ and the following assertions are equivalent: 
(a) $u(t)=0$ for $\mu$-a.e. $t \in \mathfrak{B}$ and the set $E=\left\{n \in \mathbb{N}: u\left(\mathfrak{A}_{n}\right) \neq 0\right\}$ is finite.

(b) $M_{u}$ has finite rank.

(c) $M_{u}$ has closed-range.

Proof Let $u \in L^{\Phi_{3}}(\Omega)$ and $f \in L^{\Phi_{1}}(\Omega)$, i.e. there are constants $\lambda, \alpha>0$ such that $I_{\Phi_{3}}(\lambda u)<\infty$ and $I_{\Phi_{1}}(\alpha f)<\infty$. Therefore, by the inequality $\Phi_{2}(x y) \leq \Phi_{1}(x)+$ $\Phi_{3}(y)(x, y \geq 0)$, we have

$$
I_{\Phi_{2}}(\alpha \lambda u f) \leq I_{\Phi_{1}}(\alpha f)+I_{\Phi_{3}}(\lambda u)<\infty
$$

which implies that $u f \in L^{\Phi_{2}}(\Omega)$.

Let $S$ be the support of $u$. We may assume that $\mu(S)>0$ since otherwise $M_{u}$ is a zero operator and there is nothing to prove.

We prove the implication (a) $\Rightarrow$ (b). Assume that (a) holds. Hence there is $r \in \mathbb{N}$ such that

$$
S=\bigcup_{n \in E} \mathfrak{A}_{n}=\mathfrak{A}_{n_{1}} \cup \cdots \cup \mathfrak{A}_{n_{r}}
$$

Since $\Phi_{2} \in \Delta_{2}$, the set $\left\{\chi_{\mathfrak{A}_{n_{1}}}, \ldots, \chi_{\mathfrak{A}_{n_{r}}}\right\}$ of characteristic functions generates the subspace

$$
\left\{g \in L^{\Phi_{2}}(\Omega): g(x)=0 \text { for } \mu \text { - a.e. } \mathrm{t} \in \Omega \backslash S\right\} \cong L^{\Phi_{2}}(S) \text {. }
$$

The range of $M_{u}$ is contained in the $r$-dimensional subspace $L^{\Phi_{2}}(S)$, hence $M_{u}$ has finite rank.

(b) $\Rightarrow$ (c) . If the range of the operator $M_{u}$ in $L^{\Phi_{2}}(\Omega)$ is finite-dimensional, then it is also closed, as any finite-dimensional subspace of a Banach space is a closed subspace of this space.

(c) $\Rightarrow$ (a) . Let $M_{u}$ have closed-range and assume that $\mu\{t \in \mathfrak{B}: u(t) \neq 0\}>0$. Then there is $\delta>0$ such that the set $G=\{t \in \mathfrak{B}: u(t) \geq \delta\}$ has positive measure. It is easy to see that the restriction $u_{\left.\right|_{G}}$ induces a bounded multiplication operator $M_{u_{\mid}}$from $L^{\Phi_{1}}(G)$ into $L^{\Phi_{2}}(G)$, and that if $M_{u}$ has closed-range, then $M_{u_{\left.\right|_{G}}}$ has closed-range as well.

We will show that $M_{u_{\left.\right|_{G}}}\left(L^{\Phi_{1}}(G)\right)=L^{\Phi_{2}}(G)$. Let $A$ be any measurable subset of $G$ with $\mu(A)<\infty$, and define the function $f_{A}:=\frac{1}{u_{l_{G}}} \chi_{A}$. We get

$$
I_{\Phi_{1}}\left(f_{A}\right)=\int_{G} \Phi_{1} \circ f_{A} d \mu=\int_{A} \Phi_{1} \circ \frac{1}{u_{\left.\right|_{G}}} d \mu \leq \Phi_{1}(1 / \delta) \mu(A)<\infty
$$

and so $f_{A} \in L^{\Phi_{1}}(G)$. Moreover, $M_{u_{\mid G}} f_{A}=\chi_{A}$, which implies that the linear space $M_{u_{\left.\right|_{G}}}\left(L^{\Phi_{1}}(G)\right)$ contains the set $\mathcal{F}$ of all linear combinations of characteristic functions of measurable subsets of $G$ with positive and finite measure. 
Now $\mathcal{F}$ is a dense subset of $L^{\Phi_{2}}(G)$ ([26, Lemma 4.1]) and, by assumption, $M_{u_{\left.\right|_{G}}}\left(L^{\Phi_{1}}(G)\right)$ is a closed subspace of $L^{\Phi_{2}}(G)$, therefore

$$
L^{\Phi_{2}}(G)=\overline{\mathcal{F}} \subset M_{u_{\mid G}}\left(L^{\Phi_{1}}(G)\right) \subset L^{\Phi_{2}}(G)
$$

and so $M_{u_{\left.\right|_{G}}}\left(L^{\Phi_{1}}(G)\right)=L^{\Phi_{2}}(G)$, as claimed.

Consequently, we can define the inverse multiplication operator

$$
M_{\frac{1}{u_{\mid G}}}: L^{\Phi_{2}}(G) \rightarrow L^{\Phi_{1}}(G), \quad M_{\frac{1}{u_{\mid G}}} f:=\frac{1}{u_{\left.\right|_{G}}} f .
$$

The operator $M_{\frac{1}{u_{\mid}}}$is bounded and, since $\Phi_{2}(x y) \leq \Phi_{1}(x)+\Phi_{3}(y) \quad(x, y \geq 0)$, we can apply Theorem 3.4 to conclude that $\frac{1}{u(t)}=0$ for $\mu$-a.e. $t \in G$, which is absurd. This contradiction shows that $u(t)=0$ for $\mu$-a.e. $t \in \mathfrak{B}$.

Next we show that the set $E=\left\{n \in \mathbb{N}: u\left(\mathfrak{A}_{n}\right) \neq 0\right\}$ is finite if $M_{u}$ has closed range. If $E=\emptyset$, we have nothing to prove. So let us assume that $E \neq \emptyset$. Define $S=\bigcup_{n \in E} \mathfrak{A}_{n}$.

Analogously as above, we can show that $M_{u_{\mid S}}\left(L^{\Phi_{1}}(S)\right)=L^{\Phi_{2}}(S)$. Indeed, let $A$ be a subset of $S$ with $\mu(A)<\infty$. Define the function $f_{A}:=\frac{1}{u_{I_{S}}} \chi_{A}$. The set $A$ having finite measure, we get

$$
I_{\Phi_{1}}\left(f_{A}\right)=\int_{A} \Phi_{1} \circ \frac{1}{u_{\left.\right|_{S}}} d \mu=\sum_{\mathfrak{A}_{\mathfrak{n}} \subset A} \Phi_{1}\left(1 / u\left(\mathfrak{A}_{\mathfrak{n}}\right)\right) \mu\left(\mathfrak{A}_{\mathfrak{n}}\right)<\infty .
$$

Hence $f_{A} \in L^{\Phi_{1}}(S)$. Since $M_{u_{\mid S}} f_{A}=\chi_{A}$, we conclude that $M_{u_{\mid S}}\left(L^{\Phi_{1}}(S)\right)$ contains the set $\ell_{f}^{0}$ of all linear combinations of characteristic functions of subsets of $S$ with positive and finite measure.

Now $\ell_{f}^{0}$ is a dense subset of $L^{\Phi_{2}}(S)$, and $M_{u_{\mid S}}\left(L^{\Phi_{1}}(S)\right)$ is a closed subspace of $L^{\Phi_{2}}(S)$, which implies that

$$
L^{\Phi_{2}}(S)=\overline{\ell_{f}^{0}} \subset M_{u_{\mid S}}\left(L^{\Phi_{1}}(S)\right) \subset L^{\Phi_{2}}(S),
$$

and so $M_{u_{\mid S}}\left(L^{\Phi_{1}}(S)\right)=L^{\Phi_{2}}(S)$.

We can thus define a bounded multiplication operator $M_{\frac{1}{u_{\mid}}}$from $L^{\Phi_{2}}(S)$ into $L^{\Phi_{1}}(S)$. Applying Theorem 3.4 to the operator $M_{\frac{1}{u_{\mid S}}}$, we obtain

$$
\sup _{n \in E} \frac{1}{u\left(\mathfrak{A}_{n}\right)} \Phi_{3}^{-1}\left(\frac{1}{\mu\left(\mathfrak{A}_{n}\right)}\right)<\infty
$$

Let $C=\sup _{n \in E} \frac{1}{u\left(\mathfrak{A}_{n}\right)} \Phi_{3}^{-1}\left(\frac{1}{\mu\left(\mathfrak{A}_{n}\right)}\right)>0$. Since $E \neq \varnothing$ and $1 \leq \Phi_{3}\left(C u\left(\mathfrak{A}_{n}\right)\right) \mu\left(\mathfrak{A}_{n}\right)$ for all $n \in E$, we have 


$$
\sum_{n \in E} 1 \leq \sum_{n \in E} \Phi_{3}\left(C u\left(\mathfrak{A}_{n}\right)\right) \mu\left(\mathfrak{A}_{n}\right)=\sum_{n \in E} \int_{\mathfrak{A}_{n}} \Phi_{3} \circ C u d \mu \leq \int_{\Omega} \Phi_{3} \circ C u d \mu<\infty
$$

where the final inequality follows from the assumption that $\Phi_{3} \in \Delta_{2}$. Thus $E$ must be finite.

In the next theorem we characterize closed-range multiplication operators $M_{u}$ : $L^{\Phi_{1}}(\Omega) \rightarrow L^{\Phi_{2}}(\Omega)$ under the condition that $\Phi_{1}(x y) \leq \Phi_{2}(x)+\Phi_{3}(y)$ for all $x, y \geq 0$.

Theorem 5.3 Let $\Phi_{1}, \Phi_{2}, \Phi_{3}$ be Young functions vanishing only at zero, taking only finite values, and such that $\Phi_{2}, \Phi_{3} \in \Delta_{2}$ and $\Phi_{1}(x y) \leq \Phi_{2}(x)+\Phi_{3}(y)$ for all $x, y \geq 0$. If $M_{u}$ is a bounded multiplication operator from $L^{\Phi_{1}}(\Omega)$ into $L^{\Phi_{2}}(\Omega)$ and $\frac{1}{u} \in \bar{L}_{+}^{\Phi_{3}}(\Omega)$, then the following statements are equivalent:

(a) The set $E=\left\{n \in \mathbb{N}: u\left(\mathfrak{A}_{n}\right) \neq 0\right\}$ is finite.

(b) $M_{u}$ has finite rank.

(c) $M_{u}$ has closed-range.

Proof By Theorem 3.4, we have that $u(t)=0$ for $\mu$-a.e. $t \in \mathfrak{B}$. The proofs of implications (a) $\Rightarrow$ (b) and (b) $\Rightarrow$ (c) are as in the proof of Theorem 5.2.

We prove the implication (c) $\Rightarrow$ (a). Using the same notation as in the proof of Theorem 5.2, let $S=\bigcup_{n \in E} \mathfrak{A}_{n}$ and $E \neq \varnothing$. Since $M_{u}: L^{\Phi_{1}}(S) \rightarrow L^{\Phi_{2}}(S)$ is bounded, by Theorem 3.4, we have

$$
\sup _{n \in E} u\left(\mathfrak{A}_{n}\right) \Phi_{3}^{-1}\left(\frac{1}{\mu\left(\mathfrak{A}_{n}\right)}\right)<\infty
$$

Let $C=\sup _{n \in E} u\left(\mathfrak{A}_{n}\right) \Phi_{3}^{-1}\left(\frac{1}{\mu\left(\mathfrak{A}_{n}\right)}\right)>0$. Since $E \neq \varnothing, 1 \leq \Phi_{3}\left(\frac{C}{u\left(\mathfrak{A}_{n}\right)}\right) \mu\left(\mathfrak{A}_{n}\right)$ for all $n \in E$, and $\Phi_{3} \in \Delta_{2}$, we may write

$$
\sum_{n \in E} 1 \leq \sum_{n \in E} \Phi_{3}\left(\frac{C}{u\left(\mathfrak{A}_{n}\right)}\right) \mu\left(\mathfrak{A}_{n}\right)=\sum_{n \in E} \int_{\mathfrak{A}_{n}} \Phi_{3} \circ \frac{C}{u} d \mu \leq \int_{\Omega} \Phi_{3} \circ \frac{C}{u} d \mu<\infty
$$

Thus $E$ is finite.

Now we characterize closed-range composition operators $C_{T}: L^{\Phi_{1}}(\Omega) \rightarrow$ $L^{\Phi_{2}}(\Omega)$ under the assumption that $\Phi_{2}(x y) \leq \Phi_{1}(x)+\Phi_{3}(y)$ for all $x, y \geq 0$.

We will need an elementary lemma with an easy proof which we omit.

Lemma 5.4 Let $\Phi_{1}, \Phi_{2}$ be Young functions vanishing only at zero and taking only finite values, and let $T$ be a non-singular measurable transformation of $\Omega$ such that $C_{T}$ is a bounded composition operator from $L^{\Phi_{1}}(\Omega)$ into $L^{\Phi_{2}}(\Omega)$. If $T$ is surjective, then $C_{T}$ is injective.

Theorem 5.5 Let $\Phi_{1}, \Phi_{2}, \Phi_{3}$ be Young functions vanishing only at zero, taking only finite values, and such that $\Phi_{2} \in \nabla^{\prime} \cap \Delta_{2}, \Phi_{3} \in \Delta_{2}$, and $\Phi_{2}(x y) \leq \Phi_{1}(x)+\Phi_{3}(y)$ for 
all $x, y \geq 0$. Let $T$ be a surjective non-singular measurable transformation of $\Omega$ such that $\Phi_{2}^{-1} \circ h \in L^{\Phi_{3}}(T(\Omega))$. If $C_{T}: L^{\Phi_{1}}(\Omega) \rightarrow L^{\Phi_{2}}(\Omega)$ is a bounded composition operator, then the following assertions are equivalent:

(a) $C_{T}$ has closed-range.

(b) $h(t)=0$ for $\mu$-a.e. $t \in T(\mathfrak{B})$ and the set $\left\{n \in \mathbb{N}: \mathfrak{A}_{n} \subset T(\Omega), h\left(\mathfrak{A}_{n}\right) \neq 0\right\}$ is finite.

(c) $\mu(\mathfrak{B})=0$ and the set $\left\{n \in \mathbb{N}: \mathfrak{A}_{n} \subset T(\Omega), \mu \circ T^{-1}\left(\mathfrak{A}_{n}\right) \neq 0\right\}$ is finite.

(d) $C_{T}$ has finite rank.

Proof The implications (b) $\Rightarrow$ (c) and (d) $\Rightarrow$ (a) are obvious.

We prove the implication (a) $\Rightarrow(\mathrm{b})$. Assume that $C_{T}$ has closed-range. Since $T$ is surjective, by Lemma 5.4, $C_{T}$ is injective. It is a well-known fact that an injective operator has closed-range if and only if it is bounded away from zero (see [4], III.12.5), hence $C_{T}$ is bounded away from zero. This, combined with Lemma 4.5, yields that the multiplication operator $M_{\Phi_{2}^{-1} \circ h}$ is bounded away from zero and therefore (being also injective) has closed-range. Now an application of Theorem 5.2 shows that $\Phi_{2}^{-1} \circ$ $h(t)=0$ for $\mu$-a.e. $t \in T(\mathfrak{B})$ and the set $\left\{n \in \mathbb{N}: \mathfrak{A}_{n} \subset T(\Omega), \Phi_{2}^{-1} \circ h\left(\mathfrak{A}_{n}\right) \neq 0\right\}$ is finite. We conclude that $h(t)=0$ for $\mu$-a.e. $t \in T(\mathfrak{B})$ and the set $\left\{n \in \mathbb{N}: \mathfrak{A}_{n} \subset\right.$ $\left.T(\Omega), h\left(\mathfrak{A}_{n}\right) \neq 0\right\}$ is finite.

Finally, we prove implication (c) $\Rightarrow(d)$. Assume (c) holds. It is easy to see that the range of the operator $C_{T}$ is then contained in a subspace generated by the functions $\left\{\chi_{T^{-1}\left(\mathfrak{A}_{n}\right)}: \mathfrak{A}_{n} \subset T(\Omega), \mu \circ T^{-1}\left(\mathfrak{A}_{n}\right) \neq 0\right\}$. Since the set $\left\{n \in \mathbb{N}: \mathfrak{A}_{n} \subset T(\Omega), \mu \circ\right.$ $\left.T^{-1}\left(\mathfrak{A}_{n}\right) \neq 0\right\}$ is finite, the subspace $C_{T}\left(L^{\Phi_{1}}(\Omega)\right)$ is finite-dimensional and so $C_{T}$ has finite rank.

As an easy consequence of this theorem we state

Corollary 5.6 If $\Omega$ is non-atomic, then, under the assumptions of Theorem 5.5, there are no non-zero closed-range composition operators from $L^{\Phi_{1}}(\Omega)$ into $L^{\Phi_{2}}(\Omega)$.

In the next theorem we characterize closed-range composition operators $C_{T}$ : $L^{\Phi_{1}}(\Omega) \rightarrow L^{\Phi_{2}}(\Omega)$ in the case when $\Phi_{1}(x y) \leq \Phi_{2}(x)+\Phi_{3}(y)$ for all $x, y \geq 0$ (note that compared to Theorem 5.5 the roles of the functions $\Phi_{1}$ and $\Phi_{2}$ are reversed).

Theorem 5.7 Let $\Phi_{1}, \Phi_{2}, \Phi_{3}$ be Young functions vanishing only at zero, taking only finite values, and such that $\Phi_{2} \in \nabla^{\prime} \cap \Delta_{2}, \Phi_{3} \in \Delta_{2}$, and $\Phi_{1}(x y) \leq \Phi_{2}(x)+\Phi_{3}(y)$ for all $x, y \geq 0$. Let $T$ be a surjective non-singular measurable transformation of $\Omega$ such that $\frac{1}{\Phi_{2}^{-1} \circ h} \in L^{\Phi_{3}}(\Omega)$. If $C_{T}: L^{\Phi_{1}}(\Omega) \rightarrow L^{\Phi_{2}}(\Omega)$ is a bounded composition operator, then the following assertions are equivalent:

(a) $C_{T}$ has closed-range.

(b) The set $\left\{n \in \mathbb{N}: \mathfrak{A}_{n} \subset T(\Omega), h\left(\mathfrak{A}_{n}\right) \neq 0\right\}$ is finite.

(c) The set $\left\{n \in \mathbb{N}: \mathfrak{A}_{n} \subset T(\Omega), \mu \circ T^{-1}\left(\mathfrak{A}_{n}\right) \neq 0\right\}$ is finite.

(d) $C_{T}$ has finite rank.

Proof Using Lemma 4.5 and Theorem 5.3 and a method similar as in the proof of Theorem 5.5 proves the theorem. 
Remark 5.8 As an applications of our results we derive characterizations of bounded and closed-range multiplication and composition operators in the special case of $L^{p}$ spaces. These results recover the main theorems in [38].

1. If for $1<p<q<\infty$ the multiplication operator $M_{u}$ is bounded from $L^{p}(\Omega)$ into $L^{q}(\Omega)$, then the following assertions are equivalent:

(a) $M_{u}$ has closed-range.

(b) $M_{u}$ has finite rank.

(c) The set $\left\{n \in \mathbb{N}: u\left(\mathfrak{A}_{n}\right) \neq 0\right\}$ is finite.

2. If for $1<q<p<\infty$ the multiplication operator $M_{u}$ is bounded from $L^{p}(\Omega)$ into $L^{q}(\Omega)$, then the following assertions are equivalent:

(a) $M_{u}$ has closed-range.

(b) $M_{u}$ has finite rank.

(c) $u(t)=0$ for $\mu$-almost all $t \in \mathfrak{B}$, and the set $\left\{n \in \mathbb{N}: u\left(\mathfrak{A}_{n}\right) \neq 0\right\}$ is finite.

3. If for $1<p<q<\infty$ the composition operator $C_{T}$ is bounded from $L^{p}(\Omega)$ into $L^{q}(\Omega)$, then the following assertions are equivalent:

(a) $C_{T}$ has closed-range.

(b) $C_{T}$ has finite rank.

(c) The set $\left\{n \in \mathbb{N}: h\left(\mathfrak{A}_{n}\right) \neq 0\right\}$ is finite.

(d) The set $\left\{n \in \mathbb{N}: \mu \circ T^{-1}\left(\mathfrak{A}_{n}\right) \neq 0\right\}$ is finite.

4. If for $1<q<p<\infty$ the composition operator $C_{T}$ is bounded from $L^{p}(\Omega)$ into $L^{q}(\Omega)$, then the following assertions are equivalent:

(a) $C_{T}$ has closed-range.

(b) $C_{T}$ has finite rank.

(c) $h(t)=0$ for $\mu$-almost all $t \in \mathfrak{B}$, and the set $\left\{n \in \mathbb{N}: h\left(\mathfrak{A}_{n}\right) \neq 0\right\}$ is finite.

(d) $\mu \circ T^{-1}(\mathfrak{B})=0$ and the set $\left\{n \in \mathbb{N}: \mu \circ T^{-1}\left(\mathfrak{A}_{n}\right) \neq 0\right\}$ is finite.

\section{Simultaneously necessary and sufficient conditions for continuity of the multiplication and composition operators beween distinct Orlicz spaces}

Up to this point no conditions that would be simultaneously necessary and sufficient for the continuity of the operators $M_{u}$ and $C_{T}$ have been given; the provided necessary conditions and sufficient conditions though have the merit of being quite easy to verify for a pair or a triple of Young functions involved. In order to obtain simultaneously necessary and sufficient conditions for continuity of the operators $M_{u}$ and $C_{T}$, it is convenient to take advantage of the theory of embeddings between Musielak-Orlicz spaces. In the case of non-atomic measure spaces such conditions were presented in [3]; now we will present them for purely atomic and mixed measure spaces. However, there is a price: the simultaneously necessary and sufficient conditions for the continuity of the operators $M_{u}$ and $C_{T}$ are more complicated and not as easy to verify as those presented in the preceding part of this paper.

A function $\Phi$ acting from $\Omega \times \mathbb{R}$ into $[0, \infty)$ such that $\Phi(t,$.$) is an Orlicz func-$ tion (i.e. a finite-valued continuous Young function) for $\mu$-a.e. $t \in \Omega$ and $\Phi(., x)$ is a $\Sigma$-measurable function for every $x \in \mathbb{R}$, is called a generalized Orlicz function or a Musielak-Orlicz function. The Musielak-Orlicz space $L^{\Phi}=L^{\Phi}(\Omega, \Sigma, \mu)$ is 
the space of all (equivalence classes of) $\Sigma$-measurable functions $f: \Omega \rightarrow \mathbb{R}$ such that

$$
I_{\Phi}(k f):=\int_{\Omega} \Phi(t, k f(t)) d \mu<\infty
$$

for some $k>0$ depending on $f$. The Musielak-Orlicz space furnished with the Luxemburg norm

$$
\|f\|_{\Phi}=\inf \left\{\lambda>0: I_{\Phi}\left(\frac{f}{\lambda}\right) \leq 1\right\}
$$

is a Banach space (cf. [26,28]). It is obvious that Orlicz functions are Musielak-Orlicz functions and, consequently, Orlicz spaces are Musielak-Orlicz spaces. Moreover, an Orlicz weighted space $L_{w}^{\Phi}(\Omega)$ is a Musielak-Orlicz space $L^{\Phi_{w}}(\Omega)$ with $\Phi_{w}(t, x)=$ $\Phi(x) w(t)$ for any $t \in \Omega$ and any $x \in \mathbb{R}$.

When the measure space $(\Omega, \Sigma, \mu)$ is purely atomic with infinite number of atoms, we may restrict ourselves to counting measure. Since $(\Omega, \Sigma, \mu)$ is $\sigma$-finite, it has countably many atoms, and so, without loss of generality, it may be viewed as having the form $\left(\mathbb{N}, 2^{\mathbb{N}}, \mu_{c}\right)$, where $\mu_{c}$ is the counting measure on $2^{\mathbb{N}}$. Indeed, considering atoms as singletons, we write $\mu(\{n\})=a_{n}$ for any $n \in \mathbb{N}$, where $\left\{a_{n}\right\}_{n \in \mathbb{N}}$ is a sequence of positive real numbers. If $\ell^{0}$ denotes the space of all real sequences, then, given the Musielak-Orlicz function $\Phi=\left(\Phi_{n}\right)_{n=1}^{\infty}$, i.e. a sequence of Young functions $\Phi_{n}$, we can define on $\ell^{0}$ the absolutely convex modular

$$
I_{\Phi}(f)=\sum_{n=1}^{\infty} \Phi_{n}\left(f_{n}\right) a_{n} \quad\left(f=\left(f_{n}\right) \in \ell^{0}\right) .
$$

Defining

$$
B_{\Phi}=\left\{f \in \ell^{0}: I_{\Phi}(f) \leq 1\right\}
$$

and

$$
\ell^{\Phi}(\mathbb{N})=\left\{f \in \ell^{0}: I_{\Phi}(k f)<\infty \text { for some } k>0\right\}
$$

we easily see that $B_{\Phi}$ is an absolutely convex set in $\ell^{\Phi}(\mathbb{N})$ and that all elements of $\ell^{\Phi}(\mathbb{N})$ are absorbed by $B_{\Phi}$. Consequently, the Minkowski functional generated by $B_{\Phi}$

$$
m_{\Phi}(f):=\inf \left\{\lambda>0: f / \lambda \in B_{\Phi}\right\}
$$

is a seminorm on $\ell^{\Phi}(\mathbb{N})$. If we assume that for all $n \in \mathbb{N}$ the functions $\Phi_{n}$ are not identically equal to zero, then it is easy to prove that $m_{\Phi}(f)=0$ if and only if $f=0$. Of course,

$$
m_{\Phi}(f)=\inf \left\{\lambda>0: I_{\Phi}(f / \lambda) \leq 1\right\}
$$

This is the Luxemburg norm $\|f\|_{\Phi}=m_{\Phi}(f)$. 
Let us define a new Musielak-Orlicz function $\Psi=\left(\Psi_{n}\right)_{n=1}^{\infty}$ with

$$
\Psi_{n}(x)=\Phi_{n}(x) \mu(\{n\})=\Phi_{n}(x) a_{n},
$$

where $\{n\}$ is identified with the $n$th atom of the measure $\mu$. Then the MusielakOrlicz space generated by the original Musielak-Orlicz function $\Phi$ and the original purely atomic measure with $\mu(\{n\})=a_{n}$ is isometric to the Musielak-Orlicz space generated by the Musielak-Orlicz function $\Psi$ defined above and corresponding to counting measure with $\mu_{c}(\{n\})=1$ for all $n \in \mathbb{N}$, because of the equality

$$
I_{\Psi}\left(f, \mu_{c}\right)=\sum_{n=1}^{\infty} \Psi_{n}\left(f_{n}\right)=\sum_{n=1}^{\infty} \Phi_{n}\left(f_{n}\right) a_{n}=I_{\Phi}(f, \mu) .
$$

For these reasons Musielak-Orlicz sequence spaces, without loss of generality, may be considered (and usually are) over the counting measure space $\left(\mathbb{N}, 2^{\mathbb{N}}, \mu_{c}\right)$.

Assume that $\left(\mathbb{N}, 2^{\mathbb{N}}, \mu\right)$ is a purely atomic measure space with $\mu(\{n\})>0$ for all $n \in \mathbb{N}$. If $T: \mathbb{N} \rightarrow \mathbb{N}$ is a non-singular measurable transformation and $u: \mathbb{N} \rightarrow[0, \infty)$ a measurable function, then we can define a composition operator $C_{T}$ by

$$
C_{T} f(n):=f(T(n)) \quad\left(f \in \ell^{0}, n \in \mathbb{N}\right),
$$

and a multiplication operator $M_{u}$ by

$$
M_{u} f(n):=u(n) f(n) \quad\left(f \in \ell^{0}, n \in \mathbb{N}\right) .
$$

Given Orlicz functions $\Phi$ and $\Psi$, we can look for conditions on the triples $(\Phi, \Psi, T)$ and $(\Phi, \Psi, u)$, so that $C_{T}$ or $M_{u}$ act continuously from $\ell^{\Phi}(\mathbb{N})$ into $\ell^{\Psi}(\mathbb{N})$. Note that for every function $f$ on $\mathbb{N}$ we have

$$
\begin{aligned}
I_{\Psi}\left(C_{T} f, \mu\right) & =\sum_{n=1}^{\infty} \Psi(f(T(n))) \mu(\{n\})=\sum_{m \in T(\mathbb{N})} \sum_{s \in T^{-1}(m)} \Psi(f(m)) \mu(\{s\}) \\
& =\sum_{m \in T(\mathbb{N})} \Psi(f(m)) \sum_{s \in T^{-1}(m)} \mu(\{s\})=\sum_{m \in T(\mathbb{N})} \Psi(f(m)) \mu \circ T^{-1}(\{m\}) \\
& =I_{\Psi}\left(f_{\left.\right|_{T(\mathbb{N})}}, \mu \circ T^{-1}\right)=I_{\Psi}^{\omega}\left(f_{\left.\right|_{(\mathbb{N})}}, \mu\right)
\end{aligned}
$$

where $\omega(n)=\frac{\mu \circ T^{-1}(\{n\})}{\mu(\{n\})}$. This means, of course, that $C_{T} f \in \ell^{\Psi}(\mathbb{N})$ if and only if $f_{\left.\right|_{T(\mathbb{N})}} \in \ell_{\omega}^{\Psi}(T(\mathbb{N}))$.

Similarly, for every function $f$ on $\mathbb{N}$ we have

$$
I_{\Psi}\left(M_{u} f, \mu\right)=\sum_{n=1}^{\infty} \Psi(u(n) f(n)) \mu(\{n\})=I_{\Psi}^{u}(f, \mu),
$$


which implies that $M_{u} f \in \ell^{\Psi}(\mathbb{N})$ if and only if $f \in \ell^{\Psi_{u}}(\mathbb{N})$, where $\Psi_{u}(n, x):=$ $\Psi(u(n) x)$.

To work with purely atomic measures, we need to use the discrete version of Ishii's theorem due to Shragin [37] (see also [15,17]).

Theorem 6.1 (Shragin 1976) Let $\left(\mathbb{N}, 2^{\mathbb{N}}, \mu_{c}\right)$ be the counting measure space. Let $\Phi=$ $\left(\Phi_{n}\right)_{n=1}^{\infty}$ and $\Psi=\left(\Psi_{n}\right)_{n=1}^{\infty}$ be Musielak-Orlicz functions, and let $\ell^{\Phi}(\mathbb{N})$ and $\ell^{\Psi}(\mathbb{N})$ be the Musielak-Orlicz spaces corresponding to $\Phi, \Psi$, and the counting measure $\mu_{c}$. Then $\ell^{\Phi}(\mathbb{N}) \subset \ell^{\Psi}(\mathbb{N})$ if and only if there exist $K, \delta>0$ and a sequence of non-negative numbers $\left(c_{n}\right)_{n=1}^{\infty} \in \ell^{1}(\mathbb{N})$ such that

$$
\Psi_{n}(K x) \leq \Phi_{n}(x)+c_{n}
$$

for all $x \in \mathbb{R}$ and $n \in \mathbb{N}$ satisfying $\Phi_{n}(x) \leq \delta$.

We use Shragin's result to prove the necessity part of the following theorem.

Theorem 6.2 Let $\left(\mathbb{N}, 2^{\mathbb{N}}, \mu\right)$ be a purely atomic measure space, and let $\Phi$ and $\Psi$ be Young functions vanishing only at zero and taking only finite values. The composition operator $C_{T}$ acts continuously from $\ell^{\Phi}(\mathbb{N})$ into $\ell^{\Psi}(\mathbb{N})$ if and only if there exist $K, \delta>$ 0 and a sequence of non-negative numbers $\left(c_{n}\right)_{n=1}^{\infty} \in \ell^{1}(T(\mathbb{N}))$ such that

$$
\Psi(K x) \mu \circ T^{-1}(\{n\}) \leq \Phi(x) \mu(\{n\})+c_{n}
$$

for all $x \in \mathbb{R}$ and $n \in T(\mathbb{N})$ satisfying $\Phi(x) \mu(\{n\}) \leq \delta$.

Remark 6.3 In the preceding two theorems $\delta$ can be taken in the interval $(0,1)$.

Proof (of Theorem 6.2) Assume that there are $K>0,1>\delta>0$, and a sequence of non-negative numbers $\left(c_{n}\right)_{n=1}^{\infty} \in \ell^{1}(T(\mathbb{N}))$ such that condition (6.4) is satisfied whenever $\Phi(x) \mu(\{n\}) \leq \delta$. Let $f$ be a function in the unit sphere of $\ell^{\Phi}(\mathbb{N})$. Then $I_{\Phi}(f) \leq 1$ and further $I_{\Phi}(\delta f) \leq \delta I_{\Phi}(f) \leq \delta$, hence $\Phi(\delta f(n)) \mu(\{n\}) \leq \delta$ for all $n \in \mathbb{N}$. Now setting

$$
b:=K \delta \quad \text { and } \quad c:=\sum_{n \in T(\mathbb{N})} c_{n},
$$

by (6.4) we obtain

$$
\begin{aligned}
I_{\Psi}\left(b C_{T} f\right) & =\sum_{n=1}^{\infty} \Psi(b f(T(n))) \mu(\{n\})=\sum_{n \in T(\mathbb{N})} \Psi(b f(n)) \mu \circ T^{-1}(\{n\}) \\
& \leq \sum_{n \in T(\mathbb{N})} \Phi(\delta f(n)) \mu(\{n\})+\sum_{n \in T(\mathbb{N})} c_{n} \leq \delta \sum_{n \in T(\mathbb{N})} \Phi(f(n)) \mu(\{n\})+c \\
& \leq \delta+c
\end{aligned}
$$

Denoting $d=\max (\delta+c, 1)$, we get from this that

$$
I_{\Psi}\left(\frac{b}{d} C_{T} f\right) \leq \frac{1}{d} I_{\Psi}\left(b C_{T} f\right) \leq 1 .
$$


Hence, for all functions $f$ in the unit sphere of $\ell^{\Phi}(\mathbb{N})$ we have

$$
\left\|\frac{b}{d} C_{T} f\right\|_{\ell^{\Psi}(\mathbb{N})} \leq 1,
$$

or

$$
\left\|C_{T} f\right\|_{\ell^{\Psi}(\mathbb{N})} \leq \frac{d}{b}
$$

i.e. the composition operator $C_{T}$ acts continuously from $\ell^{\Phi}(\mathbb{N})$ into $\ell^{\Psi}(\mathbb{N})$.

Now we shall prove that condition (6.4) follows from the continuity of the composition operator $C_{T}: \ell^{\Phi}(\mathbb{N}) \rightarrow \ell^{\Psi}(\mathbb{N})$. To this end, first note that, by Shragin's result, condition (6.4) is equivalent to the inclusion $\ell^{\Phi}(T(\mathbb{N})) \subset \ell_{\omega}^{\Psi}(T(\mathbb{N}))$. Indeed, it is sufficient to plug $\Phi_{n}(x)=\Phi(x) \mu(\{n\})$ and $\Psi_{n}(x)=\Psi(x) \mu \circ T^{-1}(\{n\})$ in the inequality (6.3). Hence, we only have to show that the inclusion $\ell^{\Phi}(T(\mathbb{N})) \subset \ell_{\omega}^{\Psi}(T(\mathbb{N}))$ follows from the continuity of $C_{T}$.

Let $f \in \ell^{\Phi}(T(\mathbb{N}))$. Then $\tilde{f} \in \ell^{\Phi}(\mathbb{N})$, where $\tilde{f}$ is the extension of $f$ to $\mathbb{N}$ with zero values outside of $T(\mathbb{N})$. Since $C_{T}$ is continuous, we have $C_{T} \tilde{f} \in \ell^{\Psi}(\mathbb{N})$, but, as we noticed above, this means that $f \in \ell_{\omega}^{\Psi}(T(\mathbb{N}))$.

Remark 6.4 We could have proved the sufficiency part of Theorem 6.2 via Shragin's theorem and the fact that $\left(f \in \ell^{\Phi}(\mathbb{N}) \Longrightarrow C_{T} f \in \ell^{\Psi}(\mathbb{N})\right)$ implies the continuity of the operator $C_{T}$ since any non-negative linear operator $\left(C_{T}\right.$ is such an operator) between Banach lattices is continuous. Let $\ell^{\Phi}(T(\mathbb{N})) \subset \ell_{\omega}^{\Psi}(T(\mathbb{N}))$ [which, as we have pointed out, is equivalent to condition (6.4)] and let $f \in \ell^{\Phi}(\mathbb{N})$. Then $f_{\left.\right|_{T(\mathbb{N})}} \in \ell^{\Phi}(T(\mathbb{N}))$ and so $f_{\left.\right|_{T(\mathbb{N})}} \in \ell_{\omega}^{\Psi}(T(\mathbb{N}))$, which means that indeed $C_{T} f \in \ell^{\Psi}(\mathbb{N})$. This method of proof, however, does not provide any estimate of the norm of the operator $C_{T}$.

A similar theorem can be stated for the multiplication operator.

Theorem 6.5 Let $(\mathbb{N}, \Sigma, \mu)$ be a purely atomic measure space, and let $\Phi$ and $\Psi$ be Young functions vanishing only at zero and taking only finite values. The multiplication operator $M_{u}$ acts continuously from $\ell^{\Phi}(\mathbb{N})$ into $\ell^{\Psi}(\mathbb{N})$ if and only if there exist $K, \delta>$ 0 and a sequence of non-negative numbers $\left(c_{n}\right)_{n=1}^{\infty} \in \ell^{1}(\mathbb{N})$ such that

$$
\Psi(K u(n) x) \leq \Phi(x)+c_{n}
$$

for all $x \in \mathbb{R}$ and $n \in \mathbb{N}$ satisfying $\Phi(x) \mu(\{n\}) \leq \delta$.

Proof The proof proceeds along the same lines as the proof of Theorem 6.2, and we will omit it.

Remark 6.6 As in the proof of Theorem 6.2, the sufficiency part can also be proved via Shragin's theorem with the substitutions $\Phi_{n}(x)=\Phi(x)$ and $\Psi_{n}(x)=\Psi(u(n) x)$ in condition 6.3, which then becomes equivalent to the inclusion $\ell^{\Phi}(\mathbb{N}) \subset \ell^{\Psi_{u}}(\mathbb{N})$. 
The same techniques can be applied in all theorems in which $h_{\left.\right|_{T}(\mathfrak{B})}=0 \mu$-a.e., where $h=d\left(\mu \circ T^{-1}\right) / d \mu$, and/or $u_{\left.\right|_{\mathfrak{B}}}=0 \mu$-a.e.

In the case of a general measure space $\Omega$, the non-atomic part of $\Omega$ and the purely atomic part of $\Omega$ can be treated separately, and this allows us to state the following.

Theorem 6.7 Let $(\Omega, \Sigma, \mu)$ be a measure space, and let $\Phi$ and $\Psi$ be Young functions vanishing only at zero and assuming only finite values.

(a) Assume that a non-singular measurable transformation $T$ maps $\mathfrak{B}$ into itself and $\Omega \backslash \mathfrak{B}$ into itself. The composition operator $C_{T}$ acts continuously from $L^{\Phi}(\Omega)$ into $L^{\Psi}(\Omega)$ if and only if the following conditions are jointly satisfied:

(i) there exist a constant $K>0$, a set $A \in \Sigma \cap T(\mathfrak{B})$ with $\mu(A)=0$, and a function $g \in L_{+}^{1}(T(\mathfrak{B}))$, such that

$$
\Psi(K x) h(t) \leq \Phi(x)+g(t)
$$

for all $x \geq 0$ and all $t \in T(\mathfrak{B}) \backslash A$;

(ii) condition (6.4) (we identify $\mathbb{N}$ with $\Omega \backslash \mathfrak{B}$ ).

(b) The multiplication operator $M_{u}$ acts continuously from $L^{\Phi}(\Omega)$ into $L^{\Psi}(\Omega)$ if and only if the following conditions are jointly satisfied:

(i) there exist a constant $K>0$, a set $A \in \Sigma \cap \mathfrak{B}$ with $\mu(A)=0$, and a function $g \in L_{+}^{1}(\mathfrak{B})$, such that

$$
\Psi(u(t) x) \leq \Phi(x)+g(t)
$$

for all $x \geq 0$ and all $t \in \mathfrak{B} \backslash A$;

(ii) condition (6.5) (again, we identify $\mathbb{N}$ with $\Omega \backslash \mathfrak{B}$ ).

Proof We give the proof for both operators concurrently. We can represent $L^{\Phi}(\Omega)$ and $L^{\Psi}(\Omega)$ as the direct sums of the spaces over the non-atomic part of $\Omega$ and the purely atomic part of $\Omega$, i.e. $L^{\Phi}(\Omega)=L^{\Phi}(\mathfrak{B}) \oplus L^{\Phi}(\Omega \backslash \mathfrak{B})$ and $L^{\Psi}(\Omega)=L^{\Psi}(\mathfrak{B}) \oplus$ $L^{\Psi}(\Omega \backslash \mathfrak{B})$. Therefore, every function $f \in L^{\Phi}(\Omega)$ can be uniquely written as $f=$ $f \chi_{\mathfrak{B}}+f \chi_{\Omega \backslash \mathfrak{B}}$, and we have

$$
\begin{aligned}
C_{T} f(t) & =C_{T}\left(f \chi_{\mathfrak{B}}+f \chi_{\Omega \backslash \mathfrak{B}}\right)(t) \\
& =\left(f \chi_{\mathfrak{B}}+f \chi_{\Omega \backslash \mathfrak{B}}\right)(T(t)) \\
& =f(T(t)) \chi_{T^{-1}(\mathfrak{B})}(t)+f(T(t)) \chi_{T^{-1}(\Omega \backslash \mathfrak{B})}(t) \\
& =f(T(t)) \chi_{\mathfrak{B}}(t)+f(\chi(t)) \chi_{\Omega \backslash \mathfrak{B}}(t)
\end{aligned}
$$

and

$$
\begin{aligned}
M_{u} f(t) & =M_{u}\left(f \chi_{\mathfrak{B}}+f \chi_{\Omega \backslash \mathfrak{B}}\right)(t)=u\left(f \chi_{\mathfrak{B}}+f \chi_{\Omega \backslash \mathfrak{B}}\right)(t) \\
& =u(t) f(t) \chi_{\mathfrak{B}}(t)+u(t) f(t) \chi_{\Omega \backslash \mathfrak{B}}(t) .
\end{aligned}
$$


Hence, if we define the operators $C_{T_{\left.\right|_{\mathfrak{B}}}}, M_{u_{\left.\right|_{\mathfrak{B}}}}$ on $L^{\Phi}(\mathfrak{B})$ and the operators $C_{T_{\left.\right|_{\Omega} \mid \mathfrak{B}}}$, $M_{u_{\left.\right|_{\Omega \backslash \mathfrak{B}}}}$ on $L^{\Phi}(\Omega \backslash \mathfrak{B})$ by the obvious formulas, we are justified to write

$$
C_{T}=C_{T_{\left.\right|_{\mathfrak{B}}}} \oplus C_{T_{\left.\right|_{\Omega} \mid \mathfrak{B}}} \text { and } M_{u}=M_{u_{\left.\right|_{\mathfrak{B}}}} \oplus M_{u_{\left.\right|_{\Omega} \mid \mathfrak{B}}}
$$

Of course, we have the following inequalities for the norms

$$
\begin{gathered}
\max \left\{\left\|C_{T_{\left.\right|_{\mathfrak{B}}}}\right\|,\left\|C_{T_{\left.\right|_{\Omega \backslash \mathfrak{B}}}}\right\|\right\} \leq\left\|C_{T}\right\| \leq\left\|C_{T_{\left.\right|_{\mathfrak{B}}}}\right\|+\left\|C_{T_{\left.\right|_{\Omega \backslash \mathfrak{B}}}}\right\| \\
\max \left\{\left\|M_{u_{\left.\right|_{\mathfrak{B}}}}\right\|,\left\|M_{u_{\left.\right|_{\Omega} \backslash \mathfrak{B}}}\right\|\right\} \leq\left\|M_{u}\right\| \leq\left\|M_{u_{\left.\right|_{\mathfrak{B}}}}\right\|+\left\|M_{u_{\left.\right|_{\Omega} \backslash \mathfrak{B}}}\right\| .
\end{gathered}
$$

Hence it follows that the operator $C_{T}$ acts continuously from $L^{\Phi}(\Omega)$ into $L^{\Psi}(\Omega)$ if and only if the operator $C_{T_{\left.\right|_{\mathfrak{B}}}}$ acts continuously from $L^{\Phi}(\mathfrak{B})$ into $L^{\Psi}(\mathfrak{B})$ and the operator $C_{T_{\left.\right|_{\Omega \backslash \mathfrak{B}}}}$ acts continuously from $L^{\Phi}(\Omega \backslash \mathfrak{B})$ into $L^{\Psi}(\Omega \backslash \mathfrak{B})$. The same applies to the multiplication operator: $M_{u}$ acts continuously from $L^{\Phi}(\Omega)$ into $L^{\Psi}(\Omega)$ if and only if $M_{u_{\left.\right|_{\mathfrak{B}}}}$ acts continuously from $L^{\Phi}(\mathfrak{B})$ into $L^{\Psi}(\mathfrak{B})$ and $M_{u_{\left.\right|_{\Omega} \backslash \mathfrak{B}}}$ acts continuously from $L^{\Phi}(\Omega \backslash \mathfrak{B})$ into $L^{\Psi}(\Omega \backslash \mathfrak{B})$. Therefore, the condition for boundedness of the operator $C_{T}$ is exactly the conjunction of the conditions for the boundedness of the operators $C_{T_{\mid \mathfrak{B}}}$ and $C_{T_{\left.\right|_{\Omega} \mid \mathfrak{B}}}$. Analogously, the condition for boundedness of the operator $M_{u}$ is the conjunction of conditions for the boundedness of the operators $M_{u_{\mid \mathfrak{B}}}$ and $M_{u_{\left.\right|_{\Omega} \backslash \mathfrak{B}}}$. Recall that in [3] it was shown that (a)(i) is a sufficient condition for the continuity of $C_{T}$ and that (b)(i) is a necessary and sufficient condition for the continuity of $M_{u}$ in the case of a non-atomic measure space. By analogous arguments to the ones we have used in the proof of the necessity part of Theorem 6.2 (applying Ishii's theorem rather than Shragin's) it can be shown that (a)(i) is also a necessary condition for the continuity of $C_{T}$ in the case of a non-atomic measure space, which generalizes [3], where (a)(i) was shown to be a necessary condition for the continuity of $C_{T}$ in the non-atomic case under the additional assumption that the mapping $T$ is surjective up to sets of measure zero. Moreover, Theorems 6.2 and 6.5 provide parallel sufficient and necessary conditions (a)(ii) and (b)(ii) in the case of a purely atomic measure space. This completes the proof of Theorem 6.7

Acknowledgements The authors wish to thank the anonymous referee for providing important corrections and insightful suggestions that helped to improve the text significantly.

Open Access This article is distributed under the terms of the Creative Commons Attribution 4.0 International License (http://creativecommons.org/licenses/by/4.0/), which permits unrestricted use, distribution, and reproduction in any medium, provided you give appropriate credit to the original author(s) and the source, provide a link to the Creative Commons license, and indicate if changes were made.

\section{References}

1. Ando, T.: On products of Orlicz spaces. Math. Ann. 140, 174-186 (1960)

2. Banach, S.: Théorie des opérations linéaires. Monografie Matematyczne, Warsaw (1932) 
3. Chawziuk, T., Cui, Y., Estaremi, Y., Hudzik, H., Kaczmarek, R.: Composition and multiplication operators between Orlicz function spaces. J. Inequal. Appl. 2016, 52 (2016). doi:10.1186/ s13660-016-0972-9

4. Conway, J.B.: A course in functional analysis. Springer, New York (1990)

5. Cowen, C.C., MacCluer, B.D.: Composition Operators on Spaces of Analytic Functions. Studies in Advanced Mathematics. CRC Press, Boca Raton (1995)

6. Cui, Y., Hudzik, H., Kumar, R., Maligranda, L.: Composition operators in Orlicz spaces. J. Aust. Math. Soc. 76, 189-206 (2004)

7. Cui, Y., Hudzik, H., Kumar, R., Kumar, R.: Compactness and essential norms of composition operators on Orlicz-Lorentz spaces. Appl. Math. Lett. 25(11), 1778-1783 (2012)

8. Cui, Y., Hudzik, H., Kumar, R., Kumar, R.: Erratum and addendum to the paper Compactness and essential norms of composition operators on Orlicz-Lorentz spaces [Applied Mathematics Letters 25 (2012) 1778-1783]. Comment. Math. 54(2), 259-260 (2014)

9. del Campo, R., Fernandez, A., Ferrando, I., Mayoral, F., Naranjo, F.: Multiplication operators on spaces on integrable functions with respect to a vector measure. J. Math. Anal. Appl. 343, 514-524 (2008)

10. Domański, P., Langenbruch, M.: Composition operators on spaces of real analytic functions. Math. Nachr. 254(255), 68-86 (2003)

11. Eryilmaz, I.: Weighted composition operators on weighted Lorentz spaces. Colloq. Math. 128, 143-151 (2012)

12. Estaremi, Y.: Multiplication and composition operators between two diferent Orlicz spaces. arXiv:1301.4830v2. 20 Oct 2013

13. Gupta, S., Komal, B.S., Suri, N.: Weighted composition operators on Orlicz spaces. Int. J. Contemp. Math. Sci. 1, 11-20 (2010)

14. Hencl, S., Kleprlík, L., Malý, J.: Composition operator and Sobolev-Lorentz spaces $W L^{n, q}$. Studia Math. 221, 197-208 (2014)

15. Hudzik, H., Kamińska, A.: On uniformly convexifiable and B-convex Musielak-Orlicz spaces. Comment. Math. Prace Mat. 25(1), 59-75 (1985)

16. Hudzik, H., Krbec, M.: On non-effective weights in Orlicz spaces. Indag. Mathem. (N.S.) 18(2), 215231 (2007)

17. Ishii, J.: On equivalence of modular function spaces. Proc. Jpn. Acad. 35, 551-556 (1959)

18. Jabbarzadeh, M.R.: Weighted composition operators between $L^{p}$-spaces. Bull. Korean Math. Soc. 42, 369-378 (2005)

19. Kolwicz, P., Leśnik, K., Maligranda, L.: Pointwise multipliers of Calderón-Lozanovskiĭ spaces. Math. Nachr. 286, 876-907 (2013)

20. Kolwicz, P., Leśnik, K., Maligranda, L.: Pointwise product of some Banach function spaces and factorization. J. Funct. Anal. 266, 616-659 (2014)

21. Komal, B.S., Gupta, S.: Multiplication operators between Orlicz spaces. Integr. Equ. Oper. Theory 41, 324-330 (2001)

22. Krasnoselskii, M.A., Rutickii, B.: Convex functions and Orlicz spaces. Noordhoff, Netherlands (1961) [Translated from Russian]

23. Kumar, R.: Weighted composition operators between two $L^{p}$-spaces. Mat. Vesnik 61, 111-118 (2009)

24. Kumar, R.: Composition operators on Orlicz spaces. Integr. Equ. Oper. Theory 29, 17-22 (1997)

25. Kumar, R., Kumar, R.: Composition operators on Orlicz-Lorentz spaces. Integr. Equ. Oper. Theory 60, 79-88 (2008)

26. Maligranda, L.: Orlicz spaces and interpolation, Seminars in Math. 5, Univ. Estadual de Campinas, Campinas SP, Brazil (1989)

27. Maligranda, L., Persson, L.E.: Generalized duality of some Banach function spaces. Indag. Math. 92(3), 323-338 (1989)

28. Musielak, J.: Orlicz spaces and modular spaces. Lecture Notes in Math, vol. 1034. Springer, Berlin (1983)

29. Nielsen, O.A.: An introduction to integration and measure theory. Wiley-Interscience, New York (1997)

30. Nowak, M.: On inclusions between Orlicz spaces. Comment. Math. Prace Mat. 26, 265-277 (1986)

31. Nowak, M.: Some equalities among Orlicz spaces. I. Comment. Math. Prace Mat. 29, 255-275 (1990)

32. O’Neil, R.: Fractional integration in Orlicz spaces. I. Trans. Am. Math. Soc. 115, 300-328 (1965)

33. Rao, M.M.: Convolutions of vector fields II: random walk models. Nonlinear Anal. 47, 3599-3615 (2001)

34. Rao, M.M., Ren, Z.D.: Theory of Orlicz spaces. Marcel Dekker, New York (1991) 
35. Shapiro, J.H.: Composition Operators and Classical Function Theory. Universitext Tracts in Mathematics. Springer, New York (1993)

36. Singh, R.K., Manhas, J.S.: Composition operators on function spaces, North-Holland Mathematics Studies, 179. North-Holland Publishing Co., Amsterdam (1993)

37. Shragin, I.V.: The conditions on embeddings of some classes and conclusions from them. Mat. Zam. 20, 681-692 (1976). (in Russian)

38. Takagi, H., Yokouchi, K.: Multiplication and composition operators between two $L^{p}$-spaces. Contemp. Math. 232, 321-338 (1999) 